\title{
MULTILINEAR MARCINKIEWICZ-ZYGMUND INEQUALITIES
}

\author{
DANIEL CARANDO, MARTIN MAZZITELLI, AND SHELDY OMBROSI
}

\begin{abstract}
We extend to the multilinear setting classical inequalities of Marcinkiewicz and Zygmund on $\ell^{r}$-valued extensions of linear operators. We show that for certain $1 \leq$ $p, q_{1}, \ldots, q_{m}, r \leq \infty$, there is a constant $C \geq 0$ such that for every bounded multilinear operator $T: L^{q_{1}}\left(\mu_{1}\right) \times \cdots \times L^{q_{m}}\left(\mu_{m}\right) \rightarrow L^{p}(\nu)$ and functions $\left\{f_{k_{1}}^{1}\right\}_{k_{1}=1}^{n_{1}} \subset L^{q_{1}}\left(\mu_{1}\right), \ldots,\left\{f_{k_{m}}^{m}\right\}_{k_{m}=1}^{n_{m}} \subset$ $L^{q_{m}}\left(\mu_{m}\right)$, the following inequality holds

$$
\left\|\left(\sum_{k_{1}, \ldots, k_{m}}\left|T\left(f_{k_{1}}^{1}, \ldots, f_{k_{m}}^{m}\right)\right|^{r}\right)^{1 / r}\right\|_{L^{p}(\nu)} \leq C\|T\| \prod_{i=1}^{m}\left\|\left(\sum_{k_{i}=1}^{n_{i}}\left|f_{k_{i}}^{i}\right|^{r}\right)^{1 / r}\right\|_{L^{q_{i}\left(\mu_{i}\right)}} .
$$

In some cases we also calculate the best constant $C \geq 0$ satisfying the previous inequality. We apply these results to obtain weighted vector-valued inequalities for multilinear Calderón-Zygmund operators.
\end{abstract}

\section{INTRODUCTION AND MAIN RESULTS}

The study of vector-valued inequalities for linear operators has its origins in the thirties, with works of Bochner, Marcinkiewickz, Paley and Zygmund among others. In this context we find the so-called Marcinkiewicz-Zygmund inequalities for linear operators, regarding the $\ell^{r}$-valued extensions of linear operators between real $L^{p}$-spaces. That is, given $1 \leq p, q, r \leq$ $\infty$, the triple $(p, q, r)$ is said to satisfy a Marcinkiewicz-Zygmund inequality if there is a constant $C$ such that for each bounded operator $T: L^{q}(\mu) \rightarrow L^{p}(\nu)$, ( $\mu$ and $\nu$ arbitrary $\sigma$ finite measures, $L^{q}(\mu)$ and $L^{p}(\nu)$ real spaces), each $n \in \mathbb{N}$ and functions $f_{1}, \ldots, f_{n} \in L^{q}(\mu)$,

$$
\left(\int\left(\sum_{k=1}^{n}\left|T\left(f_{k}\right)(\omega)\right|^{r}\right)^{p / r} d \nu(\omega)\right)^{1 / p} \leq C\|T\|\left(\int\left(\sum_{k=1}^{n}\left|f_{k}(\omega)\right|^{r}\right)^{q / r} d \mu(\omega)\right)^{1 / q} .
$$

The infimum of all the constants $C \geq 1$ satisfying (22) is denoted by $k_{q, p}(r)$ (setting $k_{q, p}(r)=$ $\infty$ if there is not such constant). Fixed $n \in \mathbb{N}$, let $k_{q, p}^{(n)}(r) \in[1, \infty)$ be the infimum of all the constants $C \geq 0$ satisfying (2) for each $T$ but for only $n$ functions $f_{k}$. Note that given

2010 Mathematics Subject Classification. Primary: 47H60, 47A63. Secondary: 42B20.

Key words and phrases. Vector-valued inequalities, multilinear operators, Calderón-Zygmund operators.

This project was supported in part by CONICET PIP 11220130100329CO, ANPCyT PICT 2015-2299 and UBACyT 20020130100474. The second author has a postdoctoral position from CONICET. 
$T: L^{q}(\mu) \rightarrow L^{p}(\nu)$ we can consider the natural $\ell_{n}^{r}$-valued extension $T^{\ell_{n}^{r}}: L^{q}\left(\mu, \ell_{n}^{r}\right) \rightarrow L^{p}\left(\nu, \ell_{n}^{r}\right)$ defined by

$$
T^{\ell_{n}^{r}}\left(\left(f^{1}, \ldots, f^{n}\right)\right):=\left(T f^{1}, \ldots, T f^{n}\right) .
$$

It is clear that $k_{q, p}^{(n)}(r)=\sup \left\|T^{\ell_{n}^{r}}\right\|$, where the supremum is taken over all the operators $T: L^{q}(\mu) \rightarrow L^{p}(\nu)$ with norm $\|T\| \leq 1$. Analogously, the validity of (2) is equivalent to saying that each $T: L^{q}(\mu) \rightarrow L^{p}(\nu)$ has a natural $\ell^{r}$-valued extension and, in that case, $k_{q, p}(r)=\sup \left\|T^{\ell^{r}}: L^{q}\left(\mu, \ell^{r}\right) \rightarrow L^{p}\left(\nu, \ell^{r}\right)\right\|$, where the supremum is taken over all the operators $T: L^{q}(\mu) \rightarrow L^{p}(\nu)$ with norm $\|T\| \leq 1$. It is known (see [11, 29.12]) that this supremum does not change if it is taken over two fixed measures $\mu$ and $\nu$ such that $L^{q}(\mu)$ and $L^{p}(\nu)$ are infinite-dimensional. It is worth mentioning that the problem of determining the constants $k_{q, p}^{(n)}(r)$ is a generalization of the complexification problem, that is, the computation of the so-called complexification constants $\left(k_{q, p}^{(2)}(2)\right.$ in our terminology) of operators in real $L_{p}$-spaces, which relate the norm of an operator and its complexification.

In [24], Marcinkiewicz and Zygmund proved that $k_{q, p}(2)<\infty$ for $1 \leq p, q<\infty$ and $k_{q, p}(r)<\infty$ whenever $1 \leq \max (p, q)<r<2$. In these cases, they also obtained estimates for the constants $k_{q, p}(r)$ in terms of the $q$-th moment of $r$-stable Lévy measures. In the particular case $q=p$, they obtained $k_{p, p}(2)=1$. Herz extended this last equality in [21], showing that $k_{p, p}(r)=1$ for $1<p<\infty$ and $\min (p, 2) \leq r \leq \max (p, 2)$, and Grothendieck established the important case $K_{G, \mathbb{R}}:=k_{\infty, 1}(2)<\infty\left(K_{G, \mathbb{R}}\right.$ stands for the so-called Grothendieck constant in the real case). A systematic study of the constants $k_{q, p}(r)$ and the precise asymptotic growth of $k_{q, p}^{(n)}(r)$ is addressed in [12, 17]. In the following theorem, we state some properties of the constants $k_{q, p}(r)$ (including monotonicity and duality) that can be found in the mentioned papers.

Theorem 1.1 [12, 17]. $\quad$ (i) $k_{q, p}(r)=\lim _{n \rightarrow \infty} k_{q, p}^{(n)}(r)$.

(ii) $k_{q_{1}, p_{1}}^{(n)}(r) \leq k_{q_{2}, p_{2}}^{(n)}(r)$ whenever $q_{1} \leq q_{2}$ and $p_{2} \leq p_{1}$.

(iii) As a function of $r, k_{q, p}(r)$ is decreasing on $[1,2]$ and increasing on $[2, \infty]$.

(iv) $k_{q, p}(r)=k_{p^{\prime}, q^{\prime}}\left(r^{\prime}\right)$.

In [12], the set of all the triples $1 \leq p, q, r \leq \infty$ satisfying $k_{q, p}(r)<\infty$ is determined and also the exact value of this constant is obtained in almost all the cases. We state as a theorem these results, that can be found in Sections 4, 5 and 6 of the aforementioned article.

Theorem $1.2\left[12\right.$. (i) If $q=1$ or $p=\infty$, then $k_{q, p}(r)=1$. 
(ii) Let $1<q \leq p<\infty$. Then $k_{q, p}(r)<\infty$ if and only if $\min \{q, 2\} \leq r \leq \max \{p, 2\}$. Moreover, $k_{q, p}(r)=1$ in that case.

(iii) Let $1 \leq p \leq q \leq \infty$ with $q \neq 1$ and $p \neq \infty$ (these cases are considered in (i)). Then $k_{q, p}(r)<\infty$ if and only if one of the following cases holds:

- $p=q=r$, in which case $k_{q, p}(r)=1$;

- $1 \leq p \leq q \leq 2$ and $q<r \leq 2$, in which case $k_{q, p}(r)=\frac{c_{r, q}}{c_{r, p}}$;

- $2 \leq p \leq q \leq \infty$ and $2 \leq r<p$, in which case $k_{q, p}(r)=\frac{c_{r^{\prime}, p^{\prime}}}{c_{r^{\prime}, q^{\prime}}}$

- $1 \leq p \leq 2 \leq q \leq \infty$ and $r=2$; if, moreover, $p=2$ or $q=2$ then $k_{q, p}(2)=\frac{c_{2, q}}{c_{2, p}}$.

The constant $c_{r, q}$ denotes the $q$-th moment of $r$-stable Lévy measure. The only case in which $k_{q, p}(r)$ is not determined is when $1 \leq p<r=2<q \leq \infty$.

We are interested in the study of Marcinkiewickz-Zygmund inequalities in the context of multilinear operators. In what follows, given $\left\{f_{k}\right\}_{k} \subset L^{p}(\nu)$, we denote

$$
\left\|\left(\sum_{k}\left|f_{k}\right|^{r}\right)^{1 / r}\right\|_{L^{p}(\nu)}:=\left(\int\left(\sum_{k}\left|f_{k}(\omega)\right|^{r}\right)^{p / r} d \nu(\omega)\right)^{1 / p} .
$$

Let $m \in \mathbb{N}, 1 \leq q_{1}, \ldots, q_{m}, p, r \leq \infty$ and consider $\vec{q}=\left(q_{1}, \ldots, q_{m}\right)$. We say that the triple $(p ; \vec{q} ; r)$ satisfies the Marcinkiewicz-Zygmund inequality if there is a constant $C$ such that for all bounded multilinear operators $T: L^{q_{1}}\left(\mu_{1}\right) \times \cdots \times L^{q_{m}}\left(\mu_{m}\right) \rightarrow L^{p}(\nu)$ and functions $\left\{f_{k_{1}}^{1}\right\}_{k_{1}=1}^{n_{1}} \subset L^{q_{1}}\left(\mu_{1}\right), \ldots,\left\{f_{k_{m}}^{m}\right\}_{k_{m}=1}^{n_{m}} \subset L^{q_{m}}\left(\mu_{m}\right)$, the following inequality holds (with the usual modification when $r=\infty$ )

$$
\left\|\left(\sum_{k_{1}, \ldots, k_{m}}\left|T\left(f_{k_{1}}^{1}, \ldots, f_{k_{m}}^{m}\right)\right|^{r}\right)^{1 / r}\right\|_{L^{p}(\nu)} \leq C\|T\| \prod_{i=1}^{m}\left\|\left(\sum_{k_{i}=1}^{n_{i}}\left|f_{k_{i}}^{i}\right|^{r}\right)^{1 / r}\right\|_{L^{q_{i}\left(\mu_{i}\right)}} .
$$

As in the linear case, we denote by $k_{\vec{q}, p}(r)$ the infimum of all the $C \geq 1$ satisfying (44) and we put $k_{\vec{q}, p}(r)=\infty$ when there is no such constant. We denote by $k_{q, p}^{(n)}(r) \in[1, \infty)$ the infimum of all the constants $C \geq 0$ satisfying (41) only for $n_{1}=\cdots=n_{m}=n$. It is easy to see that $\lim _{n \rightarrow \infty} k_{\vec{q}, p}^{(n)}(r)=k_{\vec{q}, p}(r)$. As observed in (3) for linear operators, the validity of (4) is equivalent to saying that each $T: L^{q_{1}}\left(\mu_{1}\right) \times \cdots \times L^{q_{m}}\left(\mu_{m}\right) \rightarrow L^{p}(\nu)$ has the $\ell^{r}$-valued extension $T^{\ell^{r}}: L^{q_{1}}\left(\mu_{1}, \ell^{r}\right) \times \cdots \times L^{q_{m}}\left(\mu_{m}, \ell^{r}\right) \rightarrow L^{p}\left(\nu, \ell^{r}(\mathbb{N} \times \cdots \times \mathbb{N})\right)$ defined by

$$
T^{\ell^{r}}\left(\left(f_{k_{1}}^{1}\right)_{k_{1}}, \ldots,\left(f_{k_{m}}^{m}\right)_{k_{m}}\right)=\left(T\left(f_{k_{1}}^{1}, \ldots, f_{k_{m}}^{m}\right)\right)_{k_{1}, \ldots, k_{m}} .
$$

In that case $k_{\vec{q}, p}(r)=\sup \left\|T^{\ell^{r}}\right\|$, where the supremum is taken over all the multilinear operators $T: L^{q_{1}}\left(\mu_{1}\right) \times \cdots \times L^{q_{m}}\left(\mu_{m}\right) \rightarrow L^{p}(\nu)$ (and over all measures $\mu_{i}$ and $\nu$ ) with norm $\|T\| \leq 1$. 
As we will point out in Section 5.1.1, it is worth mentioning that, when dealing with inequalities of the form

$$
\left\|\left(\sum_{k_{1}, \ldots, k_{m}}\left|T\left(f_{k_{1}}^{1}, \ldots, f_{k_{m}}^{m}\right)\right|^{s}\right)^{\frac{1}{s}}\right\|_{L^{p}(\nu)} \leq C \prod_{i=1}^{m}\left\|\left(\sum_{k_{i}}\left|f_{k_{i}}^{i}\right|^{r_{i}}\right)^{\frac{1}{r_{i}}}\right\|_{L^{q_{i}\left(\mu_{i}\right)}},
$$

the relation between the powers $s, r_{1}, \ldots, r_{m}$ is optimal when $s=r_{1}=\cdots=r_{m}$, as in (44). This establishes a difference with the inequalities of the form

$$
\left\|\left(\sum_{k}\left|T\left(f_{k}^{1}, \ldots, f_{k}^{m}\right)\right|^{r}\right)^{\frac{1}{r}}\right\|_{L^{p}(\nu)} \leq C \prod_{i=1}^{m}\left\|\left(\sum_{k}\left|f_{k}^{i}\right|^{r_{i}}\right)^{\frac{1}{r_{i}}}\right\|_{L^{q_{i}\left(\mu_{i}\right)}}
$$

where the sum runs over only one index $k$ and the optimal relation between the powers is given by $\frac{1}{r}=\frac{1}{r_{1}}+\cdots+\frac{1}{r_{m}}$.

In [18], in the context of vector-valued inequalities for multilinear Calderón-Zygmund operators, Grafakos and Martell addressed the multilinear Marcinkiewicz-Zygmund inequality in the particular case $r=2$, showing that $k_{\vec{q}, p}(2)<\infty$ for every $1 \leq q_{1}, \ldots, q_{m}, p<\infty$ (moreover, for $\left.0<q_{1}, \ldots, q_{m}, p<\infty\right)$. They also proved the analogous inequality for multilinear operators from $L^{q_{1}}\left(\mu_{1}\right) \times \cdots \times L^{q_{m}}\left(\mu_{m}\right)$ into $L^{p, \infty}(\nu)$. Independently, Bombal, Pérez-García and Villanueva proved in [5, Thm. 4.2] a multilinear Marcinkiewicz-Zygmund inequality in the case $r=2$ in the more general context of multilinear operators defined on Banach lattices. Our goal is to determine conditions on $p, \vec{q}, r$ such that the triple $(p, \vec{q}, r)$ satisfies the Marcinkiewicz-Zygmund inequality (4) and, if it is possible, to calculate the exact value of the constant $k_{\vec{q}, p}(r)$. In this sense, our main result is the following.

Theorem 1.3. Let $1 \leq q_{1}, \ldots, q_{m} \leq \infty, 1 \leq p<\infty$ and $\boldsymbol{q}=\max \left\{q_{1}, \ldots, q_{m}\right\}$.

(i) Suppose $\boldsymbol{q} \leq p$.

(ia) If $1 \leq \boldsymbol{q} \leq p \leq 2$, then $k_{\vec{q}, p}(r)<\infty$ iff $\boldsymbol{q} \leq r \leq 2$ or $\boldsymbol{q}=1$ and $1 \leq r \leq \infty$.

(ib) If $2 \leq \boldsymbol{q} \leq p$, then $k_{\vec{q}, p}(r)<\infty$ iff $2 \leq r \leq p$.

(ic) If $1 \leq \boldsymbol{q} \leq 2 \leq p$, then $k_{\vec{q}, p}(r)<\infty$ iff $\boldsymbol{q} \leq r \leq p$ or $\boldsymbol{q}=1$ and $1 \leq r \leq \infty$.

(ii) Suppose $p<\boldsymbol{q}$.

(iia) If $1 \leq p<\boldsymbol{q} \leq 2$, then $k_{\vec{q}, p}(r)<\infty$ iff $\boldsymbol{q}<r \leq 2$ or $\boldsymbol{q}=r=2$.

(iib) If $2<p<\boldsymbol{q}$, then $k_{\vec{q}, p}(r)<\infty$ implies $2 \leq r<p$. If $r=2$ then $k_{\vec{q}, p}(r)<\infty$.

(iic) If $1 \leq p \leq 2 \leq \boldsymbol{q}$, then $k_{\vec{q}, p}(r)<\infty$ iff $r=2$.

If (ia), (ic), (iia) holds or if $\boldsymbol{q} \leq r \leq p$, then $k_{\vec{q}, p}(r)=k_{q_{1}, p}(r) \cdots k_{q_{m}, p}(r)$. 
Note that the only case in which we do not get an equivalence is in (iib), where we obtain a necessary condition for $k_{\vec{q}, p}(r)<\infty$ (and the, already known, sufficient condition $r=2$ ). In Section 5 we address the case $0<p<1$ and the case of weak type estimates, which will be relevant in obtaining vector-valued estimates for multilinear singular integrals.

We also obtain some properties of the constants $k_{\vec{q}, p}(r)$ that, besides being important in the proof of the previous theorem, are interesting on their own. First, we study the relation between $k_{\vec{q}, p}(r)$ and the linear constants $k_{q_{1}, p}(r), \ldots, k_{q_{m}, p}(r)$. We get the following result which, in particular, shows that if $k_{q_{i}, p}(r)=\infty$ for some $1 \leq i \leq m$, then $k_{\vec{q}, p}(r)=\infty$.

Proposition 1.4. Let $1 \leq p, q_{1}, \ldots, q_{m}, r \leq \infty$. Then, for each $n \in \mathbb{N}$ we have

$$
k_{q_{1} ; p}^{(n)}(r) \cdots k_{q_{m} ; p}^{(n)}(r) \leq k_{\vec{q} ; p}^{(n)}(r) .
$$

Consequently, $k_{q_{1} ; p}(r) \cdots k_{q_{m} ; p}(r) \leq k_{\vec{q} ; p}(r)$. Moreover, if $p=r$ then equality holds.

We also prove the following monotonicity properties of $k_{\vec{q}, p}(r)$ as a function of $p$ and $r$, partially extending to multilinear setting the properties (ii) and (iii) of Theorem 1.1.

Proposition 1.5. Let $1 \leq q_{1}, \ldots, q_{m}, p \leq \infty$.

(i) If $1 \leq p_{2}<p_{1} \leq \infty$, then $k_{\vec{q}, p_{1}}^{(n)}(r) \leq k_{\vec{q}, p_{2}}^{(n)}(r)$ for all $1 \leq r \leq \infty$. Consequently, $k_{\vec{q}, p_{1}}(r) \leq k_{\vec{q}, p_{2}}(r)$.

(ii) If $1 \leq r<s \leq 2$, then $k_{\vec{q}, p}(s) \leq k_{\vec{q}, p}(r)$.

Although Theorem 1.3 seems to suggest that $k_{\vec{q}, p}(r)<\infty$ if and only if $k_{q_{i}, p}(r)<\infty$ for all $1 \leq i \leq m$, we establish an important difference between the Marcinkiewicz-Zygmund inequalities in the linear and multilinear cases when, for instance, we put $p=\infty$. First, as a simple consequence of the optimality in the well-known Littlewood's $4 / 3$ inequality, we see this different behavior between the linear and bilinear cases when we put $q_{1}=q_{2}=p=\infty$.

Remark 1.6. Denote $\vec{\infty}=(\infty, \infty)$. Then, although $k_{\infty, \infty}(r)=1$ for all $1 \leq r \leq \infty$ (this was stated in Theorem 1.2), we have $k_{\vec{\infty}, \infty}(r)=\infty$ whenever $1 \leq r<4 / 3$. Indeed, suppose that $k_{\vec{\infty}, \infty}(r)<\infty$. Then, for any $n \in \mathbb{N}$ and $T: \ell_{n}^{\infty} \times \ell_{n}^{\infty} \rightarrow \mathbb{R}$ we have

$$
\left(\sum_{k_{1}, k_{2}=1}^{n}\left|T\left(e_{k_{1}}, e_{k_{2}}\right)\right|^{r}\right)^{1 / r} \leq k_{\vec{\infty}, \infty}(r)\|T\| \prod_{i=1}^{2}\left\|\left(\sum_{k_{i}=1}^{n}\left|e_{k_{i}}\right|^{r}\right)^{1 / r}\right\|_{\ell_{n}^{\infty}}=k_{\vec{\infty}, \infty}(r)\|T\| .
$$

Thus, we obtain $\left(\sum_{k_{1}, k_{2}=1}^{\infty}\left|T\left(e_{k_{1}}, e_{k_{2}}\right)\right|^{r}\right)^{1 / r} \leq k_{\vec{\infty}, \infty}(r)\|T\|$ for every bilinear form $T: c_{0} \times$ $c_{0} \rightarrow \mathbb{R}$. The optimality in Littlewood's $4 / 3$ inequality implies that $r \geq 4 / 3$. 
The next proposition generalizes the previous remark showing that, in contrast to the linear case where $k_{q, \infty}(r)=1$ for all $1 \leq q, r \leq \infty$, in the $m$-linear case $k_{\vec{q}, \infty}(r)=\infty$ for appropriate choices of $m \in \mathbb{N}$ and $1 \leq q_{1}, \ldots, q_{m}, r \leq \infty$. In particular, we see that $k_{q_{i}, p}(r)<\infty$ for all $1 \leq i \leq m$ does not imply, in general, $k_{\vec{q}, p}(r)<\infty$. It should be noted that, just as in Remark 1.6 the optimality of Littlewood's 4/3 inequality was used, Proposition 1.7 is related to the optimality of some of its multilinear versions [14], such as the Bohnenblust-Hille inequality [6] (see also [10, 22]). In fact, our proof follows the spirit of the new approaches to these inequalities by means of probabilistic method [27] (see Lemma 4.1).

Proposition 1.7. Let $1 \leq q_{1}, \ldots, q_{m} \leq \infty$ and $\boldsymbol{q}=\max \left\{q_{1}, \ldots, q_{m}\right\}$. Then, for

$$
1 \leq r<m\left(\frac{1}{\max \left(\boldsymbol{q}^{\prime}, 2\right)}+\frac{1}{\min \left(q_{1}, 2\right)}+\cdots+\frac{1}{\min \left(q_{m}, 2\right)}\right)^{-1}
$$

we have $k_{\vec{q}, \infty}(r)=\infty$, while $k_{q_{i}, \infty}(r)=1,1 \leq i \leq m$.

Remark 1.8. It is easy to check that the expression in the right hand side of (7) is smaller than $\min \{\boldsymbol{q}, 2\}$. On the other hand, since $k_{\vec{q}, \infty}(\min \{\boldsymbol{q}, 2\})<\infty$ and $k_{\vec{q}, \infty}(\infty)=1$ (see Theorem 1.3 (ib) and Remark 2.3), by Corollary 2.6 below we have $k_{\vec{q}, \infty}(r)<\infty$ for $\min \{\boldsymbol{q}, 2\} \leq r \leq \infty$. This brings up the question on the behaviour of $k_{\vec{q}, \infty}(r)$ for the remaining range of values of $r$. Although the multilinear versions of Littlewood inequalities are understood for the full range of exponents, we were not be able to fill the gap for $r$ in our results.

As the nature of Marcinkiewicz-Zygmund inequalities is related to the study of $\ell^{r}$-valued extensions of bounded multilinear operators on $L^{p}$-spaces, a brief review of some of the inequalities involving Theorem 1.3 allows us to obtain vector-valued estimates for multilinear singular integrals. For instance, we prove that if $1<q_{1}, \ldots, q_{m}<r<2$ and $p>0$ are such that $\frac{1}{p}=\frac{1}{q_{1}}+\cdots+\frac{1}{q_{m}}$ and $\vec{w}=\left(w_{1}, \ldots, w_{m}\right)$ satisfies the multilinear $A_{\vec{q}}$ condition defined in [23], then there exists a constant $C>0$ such that for every Calderón-Zygmund multilinear operator $T$ we have

$$
\left\|\left(\sum_{k_{1}, \ldots, k_{m}}\left|T\left(f_{k_{1}}^{1}, \ldots, f_{k_{m}}^{m}\right)\right|^{r}\right)^{\frac{1}{r}}\right\|_{L^{p}\left(\nu_{\vec{w}}\right)} \leq C\|T\| \prod_{i=1}^{m}\left\|\left(\sum_{k_{i}}\left|f_{k_{i}}^{i}\right|^{r}\right)^{\frac{1}{r}}\right\|_{L^{q_{i}}\left(w_{i}\right)},
$$

where $\nu_{\vec{w}}=\prod_{i=1}^{m} w_{i}^{p / q_{i}}$. We address the expected weak type estimates when some of the exponents $q_{i}$ are equal to one. We compare our results with some known vector-valued estimates obtained in [1, 2, 8, 18]. 
Finally, we present the multilinear version of [16, Chapter V, Thm. 1.12], which states that if $T: L^{q}(\mu) \rightarrow L^{p}(\nu)$ is a positive linear operator (that is, $f \geq 0$ implies $T(f) \geq 0$ ), then the Marcinkiewicz-Zygmund inequality (2) holds for all $1 \leq r \leq \infty$, with $C=1$.

Proposition 1.9. Let $0<p, q_{1}, \ldots, q_{m} \leq \infty, 1 \leq r \leq \infty$ and $T: L^{q_{1}}\left(\mu_{1}\right) \times \cdots L^{q_{m}}\left(\mu_{m}\right) \rightarrow$ $L^{p}(\nu)$ be a positive multilinear operator. Then

$$
\left\|\left(\sum_{k_{1}, \ldots, k_{m}}\left|T\left(f_{k_{1}}^{1}, \ldots, f_{k_{m}}^{m}\right)\right|^{r}\right)^{1 / r}\right\|_{L^{p}(\nu)} \leq\|T\| \prod_{i=1}^{m}\left\|\left(\sum_{k_{i}=1}^{n_{i}}\left|f_{k_{i}}^{i}\right|^{r}\right)^{1 / r}\right\|_{L^{q_{i}\left(\mu_{i}\right)}}
$$

for any choice of functions $\left\{f_{k_{i}}^{i}\right\}_{k_{i}=1}^{n_{i}} \subset L^{q_{i}}\left(\mu_{i}\right), 1 \leq i \leq m$.

As an immediate consequence, we obtain the estimate

$$
\left\|\left(\sum_{k_{1}, k_{2}}\left|f_{k_{1}}^{1} * f_{k_{2}}^{2}\right|^{r}\right)^{1 / r}\right\|_{L^{p}(\mathbb{R})} \leq\left\|\left(\sum_{k_{1}=1}^{n_{1}}\left|f_{k_{1}}^{1}\right|^{r}\right)^{1 / r}\right\|_{L^{q_{1}(\mathbb{R})}}\left\|\left(\sum_{k_{2}=1}^{n_{2}}\left|f_{k_{2}}^{2}\right|^{r}\right)^{1 / r}\right\|_{L^{q_{2}(\mathbb{R})}}
$$

for the bilinear convolution operator, whenever $\frac{1}{q_{1}}+\frac{1}{q_{2}}=\frac{1}{p}+1$.

Structure of the article. The article is organized as follows. In Section 2 we prove the mentioned properties of the constants $k_{\vec{q}, p}(r)$. The inequality $k_{q_{1} ; p}(r) \cdots k_{q_{m} ; p}(r) \leq k_{\vec{q} ; p}(r)$ is almost immediate, while the equality in the case $p=r$ follows by induction. For the monotonicity of $k_{\vec{q}, p}(r)$ as a function of $p$ we use a duality argument. The monotonicity in $r$ makes use of $r$-stable Lévy measures and a generalization of some arguments in [17]. We include in this section a kind of interpolation property of $k_{\vec{q}, p}(r)$ as a function of $r$ (see Corollary 2.6) and we also prove that $k_{\vec{q}, p}(r)=1$ when $\vec{q}=(1, \ldots, 1)$. Our main results are given in Section 3, where we determine conditions on $\vec{q}, p$ and $r$ so that $k_{\vec{q}, p}(r)<\infty$ and we obtain (in many cases) the exact value of these constants. In Theorem 3.1 we focus on the case $1 \leq q_{1}, \ldots, q_{m} \leq 2,1 \leq p<\infty$. The proof of this theorem combines some classical arguments with properties from Section 2 and the values of the constants $k_{q_{i}, p}(r)$ obtained in [12. In Section 3.2 we include the proof of Theorem 1.3, which at that point is just a combination of the results previously obtained. In Section 4 we treat the case $p=\infty$, showing (see Proposition 1.7) that the behavior of $k_{\vec{q}, \infty}(r)$ is very different from that of $k_{q_{i}, \infty}(r)$. The proof relies on a multilinear version of a Kahane-Salem-Zygmund inequality. In Section 5 we discuss some applications of the Marcinkiewicz-Zygmund inequalities. We deal with weighted vector-valued inequalities for multilinear Calderón-Zygmund operators in Section 5.1 and, 
in Section 5.2, we prove Proposition 1.9, which gives vector-valued inequalities for positive multilinear operators (such as the convolution).

Notation. All the Banach spaces considered are real and all the measure spaces $(\Omega, \mu)$ are $\sigma$-finite. As usual, given a measure space $(\Omega, \mu)$ the space of measurable functions $f: \Omega \rightarrow \mathbb{R}$ such that $\int_{\Omega}|f(\omega)|^{p} d \mu(\omega)<\infty$ is denoted by $L^{p}(\mu)=L^{p}(\Omega ; d \mu)$ (we omit the set $\Omega$ which will be clear by context). Given a Banach space $X$, we denote by $L^{p}(\mu, X)$ the vector space of $X$-valued $\mu$-measurable functions $f: \Omega \rightarrow X$ such that $\|f(\cdot)\|_{X}^{p}$ is $\mu$-integrable. When $1 \leq p \leq \infty$, this is a Banach space with the natural norm $\|f\|_{L^{p}(\mu, X)}=\left(\int\|f(\omega)\|_{X}^{p} d \mu(\omega)\right)^{1 / p}$ (the case $p=\infty$ is defined analogously). Following the standard notation, we denote by $\ell^{p}$ the space of sequences $\left(a_{k}\right)_{k \in \mathbb{N}}$ in $\mathbb{R}$ such that $\left\|\left(a_{k}\right)_{k}\right\|_{\ell^{p}}=\left(\sum_{k}\left|a_{k}\right|^{p}\right)^{1 / p}<\infty$ and $\ell_{n}^{p}=\left(\mathbb{R}^{n},\|\cdot\|_{\ell^{p}}\right)$, with the usual modification when $p=\infty$.

\section{BASIC PROPERTIES OF $k_{\vec{q}, p}(r)$}

2.1. Relation between the linear and multilinear constants. Before proving Proposition 1.4 we state the following easy remark, whose proof is omitted. Recall that if $\left(\Omega_{1}, \nu_{1}\right), \ldots,\left(\Omega_{m}, \nu_{m}\right)$ are measure spaces, then $\nu_{1} \otimes \cdots \otimes \nu_{m}$ denotes the product measure on $\Omega_{1} \times \cdots \times \Omega_{m}$.

Remark 2.1. Let $T_{i}: L^{q_{i}}\left(\mu_{i}\right) \rightarrow L^{p}\left(\nu_{i}\right)$ be bounded operators $(i=1, \ldots, m)$ and let $T: L^{q_{1}}\left(\mu_{1}\right) \times$ $\cdots \times L^{q_{m}}\left(\mu_{m}\right) \rightarrow L^{p}\left(\nu_{1} \otimes \cdots \otimes \nu_{m}\right)$ be the m-linear operator defined as $T\left(f^{1}, \ldots, f^{m}\right)=$ $T_{1}\left(f^{1}\right) \otimes \cdots \otimes T_{m}\left(f^{m}\right)$, , i.e.,

$$
T\left(f^{1}, \ldots, f^{m}\right)\left(\omega_{1}, \ldots, \omega_{m}\right)=T_{1}\left(f^{1}\right)\left(\omega_{1}\right) \cdots T_{m}\left(f^{m}\right)\left(\omega_{m}\right) .
$$

Then $\|T\|=\left\|T_{1}\right\| \cdots\left\|T_{m}\right\|$ and

$$
\left\|\left(\sum_{k_{1}, \ldots, k_{m}=1}^{n}\left|T\left(f_{k_{1}}^{1}, \ldots, f_{k_{m}}^{m}\right)\right|^{r}\right)^{1 / r}\right\|_{L^{p}\left(\nu_{1} \otimes \cdots \otimes \nu_{m}\right)}=\prod_{i=1}^{m}\left\|\left(\sum_{k_{i}=1}^{n}\left|T_{i}\left(f_{k_{i}}^{i}\right)\right|^{r}\right)^{1 / r}\right\|_{L^{p}\left(\nu_{i}\right)}
$$

for each $n \in \mathbb{N}$ and $\left\{f_{k_{i}}^{i}\right\}_{k_{i}=1}^{n} \subset L^{q_{i}}\left(\mu_{i}\right)$.

Proof of Proposition 1.4. It is clear that $k_{q_{1} ; p}^{(n)}(r) \cdots k_{q_{m} ; p}^{(n)}(r)$ is the infimum of all the constants $C \geq 0$ satisfying

$$
\prod_{i=1}^{m}\left\|\left(\sum_{k_{i}=1}^{n}\left|T_{i}\left(f_{k_{i}}^{i}\right)\right|^{r}\right)^{1 / r}\right\|_{L^{p}\left(\nu_{i}\right)} \leq C \prod_{i=1}^{m}\left\|T_{i}\right\|\left\|\left(\sum_{k_{i}=1}^{n}\left|f_{k_{i}}^{i}\right|^{r}\right)^{1 / r}\right\|_{L^{q_{i}\left(\mu_{i}\right)}}
$$


for every $T_{i}: L^{q_{i}}\left(\mu_{i}\right) \rightarrow L^{p}\left(\nu_{i}\right)$ and all functions $\left\{f_{k_{i}}^{i}\right\}_{k_{i}=1}^{n} \subset L^{q_{i}}\left(\mu_{i}\right)(1 \leq i \leq m)$. By the previous remark, we see that $k_{q_{1} ; p}^{(n)}(r) \cdots k_{q_{m} ; p}^{(n)}(r)$ is the infimum of all the constants $C \geq 0$ satisfying

$$
\left\|\left(\sum_{k_{1}, \ldots, k_{m}=1}^{n}\left|T\left(f_{k_{1}}^{1}, \ldots, f_{k_{m}}^{m}\right)\right|^{r}\right)^{1 / r}\right\|_{L^{p}\left(\nu_{1} \otimes \cdots \otimes \nu_{m}\right)} \leq C\|T\| \prod_{i=1}^{m}\left\|\left(\sum_{k_{i}=1}^{n}\left|f_{k_{i}}^{i}\right|^{r}\right)^{1 / r}\right\|_{L^{q_{i}\left(\mu_{i}\right)}}
$$

for every $m$-linear operator $T: L^{q_{1}}\left(\mu_{1}\right) \times \cdots \times L^{q_{m}}\left(\mu_{m}\right) \rightarrow L^{p}\left(\nu_{1} \otimes \cdots \otimes \nu_{m}\right)$ of the form $T\left(f^{1}, \cdots, f^{m}\right)=T_{1}\left(f^{1}\right) \otimes \cdots \otimes T_{m}\left(f^{m}\right)$ and functions $\left\{f_{k_{i}}^{i}\right\}_{k_{i}=1}^{n} \subset L_{q_{i}}\left(\mu_{i}\right)$. This shows that the constant $k_{\vec{q}, p}^{(n)}(r)$, which is the infimum of all the constants satisfying (10) for every $m$-linear operator from $L^{q_{1}}\left(\mu_{1}\right) \times \cdots \times L^{q_{m}}\left(\mu_{m}\right)$ to $L^{p}(\nu)$, is greater than or equal to $k_{q_{1} ; p}^{(n)}(r) \cdots k_{q_{m} ; p}^{(n)}(r)$.

To prove equality when $p=r$, we reason by induction on $m$ (see, for instance, [5, Thm. 3.1] for a similar argument). For $m=1$ the result is trivial, then let $m \geq 2$ and suppose that the result holds for $m-1$. We write the proof for $1 \leq r<\infty$, the case $r=\infty$ being analogous. Fix $T: L^{q_{1}}\left(\mu_{1}\right) \times \cdots \times L^{q_{m}}\left(\mu_{m}\right) \rightarrow L^{r}(\nu), n \in \mathbb{N}$ and $\left\{f_{k_{i}}^{i}\right\}_{k_{i}=1}^{n} \subset L^{q_{i}}\left(\mu_{i}\right)$. Our goal is to prove

$$
\left\|\left(\sum_{k_{1}, \ldots, k_{m}}\left|T\left(f_{k_{1}}^{1}, \ldots, f_{k_{m}}^{m}\right)\right|^{r}\right)^{1 / r}\right\|_{L^{r}(\nu)} \leq k_{q_{1}, r}^{(n)}(r) \cdots k_{q_{m}, r}^{(n)}(r)\|T\| \prod_{i=1}^{m}\left\|\left(\sum_{k_{i}=1}^{n}\left|f_{k_{i}}^{i}\right|^{r}\right)^{1 / r}\right\|_{L^{q_{i}\left(\mu_{i}\right)}},
$$

which would give the remaining inequality $k_{\vec{q}, r}^{(n)}(r) \leq k_{q_{1}, r}^{(n)}(r) \cdots k_{q_{m}, r}^{(n)}(r)$. For each $2 \leq i \leq m$ let $\nu_{i}$ be the counting measure on $\mathbb{N}$ and define $S: L^{q_{1}}\left(\mu_{1}\right) \rightarrow L^{r}\left(\nu \otimes \nu_{2} \otimes \cdots \otimes \nu_{m}\right)$ by

$$
S\left(f^{1}\right)\left(\omega, k_{2}, \ldots, k_{m}\right)=\left\{\begin{array}{cl}
T\left(f^{1}, f_{k_{2}}^{2}, \ldots, f_{k_{m}}^{m}\right)(\omega) & \text { if } k_{2}, \ldots, k_{m} \leq n \\
0 & \text { otherwise }
\end{array}\right.
$$

It is easy to check that

$$
\left\|\left(\sum_{k_{1}=1}^{n}\left|S\left(f_{k_{1}}^{1}\right)\right|^{r}\right)^{1 / r}\right\|_{L^{r}\left(\nu \otimes \nu_{2} \otimes \cdots \otimes \nu_{m}\right)}=\left\|\left(\sum_{k_{1}, \ldots, k_{m}=1}^{n}\left|T\left(f_{k_{1}}^{1}, \ldots, f_{k_{m}}^{m}\right)\right|^{r}\right)^{1 / r}\right\|_{L^{r}(\nu)} .
$$

Now, on the one hand we have

$$
\left\|\left(\sum_{k_{1}=1}^{n}\left|S\left(f_{k_{1}}^{1}\right)\right|^{r}\right)^{1 / r}\right\|_{L^{r}\left(\nu \otimes \nu_{2} \otimes \cdots \otimes \nu_{m}\right)} \leq k_{q_{1}, r}^{(n)}(r)\|S\|\left\|\left(\sum_{k_{1}=1}^{n}\left|f_{k_{1}}^{1}\right|\right)^{1 / r}\right\|_{L^{q_{1}}\left(\mu_{1}\right)}
$$


and on the other hand, if we call $T_{f^{1}}\left(f^{2}, \ldots, f^{m}\right)=T\left(f^{1}, \ldots, f^{m}\right)$ and $\vec{q}_{2, \ldots, m}=\left(q_{2}, \ldots, q_{m}\right)$, then

$$
\begin{aligned}
\left\|S\left(f^{1}\right)\right\|_{L^{r}\left(\nu \otimes \nu_{2} \otimes \cdots \otimes \nu_{m}\right)} & =\left(\int \sum_{k_{2}, \ldots, k_{m}=1}^{n}\left|T_{f^{1}}\left(f_{k_{2}}^{2}, \ldots, f_{k_{m}}^{m}\right)(\omega)\right|^{r} d \nu(\omega)\right)^{1 / r} \\
& \leq k_{\vec{q}_{2}, \ldots, m, r}^{(n)}(r)\left\|T_{f^{1}}\right\| \prod_{i=2}^{m}\left\|\left(\sum_{k_{i}=1}^{n}\left|f_{k_{i}}^{i}\right|\right)^{1 / r}\right\|_{L^{q_{i}}\left(\mu_{i}\right)} \\
& \leq k_{\vec{q}_{2}, \ldots, m, r}^{(n)}(r)\|T\|\left\|f^{1}\right\|_{L^{q_{1}\left(\mu_{1}\right)}} \prod_{i=2}^{m}\left\|\left(\sum_{k_{i}=1}^{n}\left|f_{k_{i}}^{i}\right|\right)^{1 / r}\right\|_{L^{q_{i}\left(\mu_{i}\right)}},
\end{aligned}
$$

from where we deduce

$$
\|S\| \leq k_{\vec{q}_{2, \ldots, m}, r}^{(n)}(r)\|T\| \prod_{i=2}^{m}\left\|\left(\sum_{k_{i}=1}^{n}\left|f_{k_{i}}^{i}\right|\right)^{1 / r}\right\|_{L^{q_{i}\left(\mu_{i}\right)}} .
$$

Putting (11), (12) and (13) together we obtain $k_{\vec{q}, r}^{(n)}(r) \leq k_{q_{1}, r}^{(n)}(r) k_{\overrightarrow{q_{2}, \ldots, m}, r}^{(n)}(r)$. By the induction hypothesis we have $k_{\vec{q}_{2, \ldots, m}, r}^{(n)}(r) \leq k_{q_{2}, r}^{(n)}(r) \cdots k_{q_{m}, r}^{(n)}(r)$ and this proves the statement.

If $T_{1}: L^{q_{1}}\left(\mu_{1}\right) \rightarrow L^{p}\left(\nu_{1}\right)$ and $T_{2}: L^{q_{2}}\left(\mu_{2}\right) \times \cdots \times L^{q_{m}}\left(\mu_{m}\right) \rightarrow L^{p}\left(\nu_{2}\right)$, then

$$
T\left(f^{1}, \cdots, f^{m}\right)\left(\omega_{1}, \omega_{2}\right)=T_{1}\left(f^{1}\right)\left(\omega_{1}\right) T_{2}\left(f^{2}, \ldots, f^{m}\right)\left(\omega_{2}\right)
$$

is an $m$-linear operator satisfying $\|T\|=\left\|T_{1}\right\|\left\|T_{2}\right\|$ and an equality analogous to (9). Then, reasoning as in the proof of Proposition 1.4, we see that if $(\vec{q}, p, r)$ satisfies the ( $m$-linear) Marcinkiewicz-Zygmund inequality, then $\left(\left(q_{2}, \ldots, q_{m}\right), p, r\right)$ satisfies the $((m-1)$-linear $)$ Marcinkiewicz-Zygmund inequality. Clearly, the same reasoning applies with any $q_{i}$ instead of $q_{1}$. Then, we have the following.

Remark 2.2. Let $1 \leq p, q_{1}, \ldots, q_{m}, r \leq \infty$. Then,

$$
k_{q_{j}, p}(r) k_{\left(q_{2}, \ldots, q_{j-1}, q_{j+1}, \ldots, q_{m}\right), p}(r) \leq k_{\left(q_{1}, \ldots, q_{m}\right), p}(r)
$$

for $j=1, \ldots, m$.

Applying Theorem 1.2 and Proposition 1.4 for the case $p=r$, we see that $k_{\vec{q}, r}(r)<\infty$ if and only if $r=2$ or $q_{i} \leq r$ for all $1 \leq i \leq m$. Moreover, if $q_{i} \leq r$ for all $1 \leq i \leq m$ then $k_{\vec{q}, r}(r)=1$. This, together with the monotonicity in $p$ (see Proposition 1.5), gives the following result.

Remark 2.3. If $1 \leq q_{1}, \ldots, q_{m} \leq r \leq p \leq \infty$ then $k_{\vec{q}, p}(r)=1$. 
2.2. Monotonicity in $p$. Next, we prove the property of monotonicity of $k_{\vec{q}, p}(r)$ as a function of $p$ stated in Proposition 1.5 (i). The proof follows a duality argument analogous to that of [18, Thm. 6].

Proof of monotonicity in $p$. Let $T: L^{q_{1}}\left(\mu_{1}\right) \times \cdots \times L^{q_{m}}\left(\mu_{m}\right) \rightarrow L^{p_{1}}(\nu)$ be an $m$-linear operator and $\left\{f_{k_{i}}^{i}\right\}_{k_{i}=1}^{n} \subset L^{q_{i}}\left(\mu_{i}\right)$. Assume $1 \leq r<\infty$, the case $r=\infty$ being completely analogous. By duality we have

$$
\begin{aligned}
& \left\|\left(\sum_{k_{1}, \ldots, k_{m}=1}^{n}\left|T\left(f_{k_{1}}^{1}, \ldots, f_{k_{m}}^{m}\right)\right|^{r}\right)^{1 / r}\right\|_{L^{p_{1}(\nu)}} \\
& =\sup _{\|g\|_{L}\left(p_{1} / p_{2}\right)^{\prime} \leq 1}\left\|\left(\sum_{k_{1}, \ldots, k_{m}=1}^{n}\left|T\left(f_{k_{1}}^{1}, \ldots, f_{k_{m}}^{m}\right)\right|^{r}\right)^{1 / r}|g|^{1 / p_{2}}\right\|_{L^{p_{2}(\nu)}} .
\end{aligned}
$$

Now, fixed $g \in L^{\left(p_{1} / p_{2}\right)^{\prime}}(\nu)$ with norm at most 1 , consider $T_{g}: L^{q_{1}}\left(\mu_{1}\right) \times \cdots \times L^{q_{m}}\left(\mu_{m}\right) \rightarrow$ $L^{p_{2}}(\nu)$ defined by

$$
T_{g}\left(f^{1}, \ldots, f^{m}\right)=|g|^{1 / p_{2}} T\left(f^{1}, \ldots, f^{m}\right)
$$

and note that (14) gives

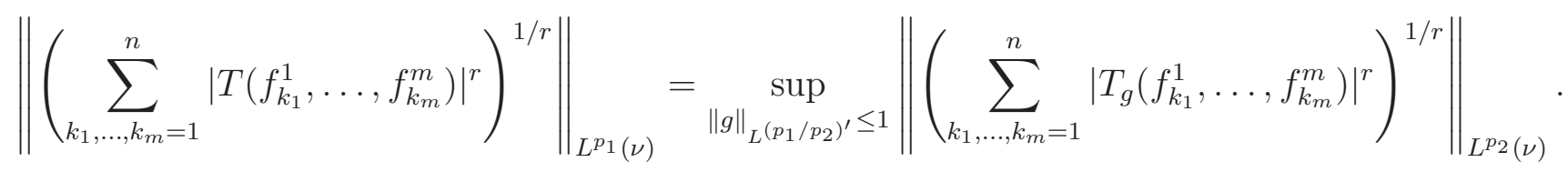

Since $\left\|T_{g}\right\| \leq\|T\|$ for every $g \in L^{\left(p_{1} / p_{2}\right)^{\prime}}(\nu)$ of norm at most 1 (just apply Hölder's inequality), we obtain

$$
\left\|\left(\sum_{k_{1}, \ldots, k_{m}=1}^{n}\left|T\left(f_{k_{1}}^{1}, \ldots, f_{k_{m}}^{m}\right)\right|^{r}\right)^{1 / r}\right\|_{L^{p_{1}(\nu)}} \leq k_{\vec{q}, p_{2}}^{(n)}(r)\|T\| \prod_{i=1}^{m}\left\|\left(\sum_{k_{i}=1}^{n}\left|f_{k_{i}}^{i}\right|^{r}\right)^{1 / r}\right\|_{L^{q_{i}\left(\mu_{i}\right)}},
$$

which gives $k_{\vec{q}, p_{1}}^{(n)}(r) \leq k_{\vec{q}, p_{2}}^{(n)}(r)$.

2.3. Monotonicity and interpolation property in $r$. For the proof of the monotonicity of $k_{\vec{q}, p}(r)$ as a function of $r$ (when $1 \leq r \leq 2$ ), we make use of $r$-stable variables (or $r$ stable Lévy measures). A random variable is said to be $r$-stable $(0<r \leq 2)$ if its Fourier transform on $\mathbb{R}$ is equal to $e^{-|x|^{r}}$. The $s$-th moment of the $r$-stable variable $w$ is given by $c_{r, s}=\left(\int_{\mathbb{R}}|t|^{s} w(t) d t\right)^{1 / s}$. Note that 2 -stable variables are just Gaussian variables. One importance of $r$-stable variables relies on the following well known property, whose proof can be found in [26, 21.1.3]: For $0<s<r<2$ or $r=2$ and $0<s<\infty$, if $\left\{w_{k}\right\}_{k}$ is a sequence 
of independent $r$-stable random variables defined on $[0,1]$, then

$$
\left(\int_{0}^{1}\left|\sum_{k=1}^{n} a_{k} w_{k}(t)\right|^{s} d t\right)^{1 / s}=c_{r, s}\left(\sum_{k=1}^{n}\left|a_{k}\right|^{r}\right)^{1 / r}
$$

for every sequence $\left\{a_{k}\right\}_{k} \subset \mathbb{R}$ and any $n \in \mathbb{N}$.

The following lemma will be a main tool to prove the desired monotonicity and will also be useful in Section 3.1.

Lemma 2.4. Let $0<r \leq 2$ and $0<p<\infty$. If $\left\{w_{k_{1}}\right\}_{k_{1}}, \ldots,\left\{w_{k_{m}}\right\}_{k_{m}}$ are sequences of mutually independent $r$-stable random variables defined on $[0,1]$, then

$$
\begin{aligned}
& C\left(\sum_{k_{1}, \ldots, k_{m}=1}^{n}\left|a_{k_{1}, \ldots, k_{m}}\right|^{r}\right)^{1 / r} \\
\leq & \left(\int_{0}^{1} \cdots \int_{0}^{1}\left|\sum_{k_{1}, \ldots, k_{m}=1}^{n} a_{k_{1}, \ldots, k_{m}} w_{k_{1}}\left(t_{1}\right) \cdots w_{k_{m}}\left(t_{m}\right)\right|^{p} d t_{1} \cdots d t_{m}\right)^{1 / p}
\end{aligned}
$$

for every sequence $\left\{a_{k_{1}, \ldots, k_{m}}\right\}_{k_{1}, \ldots, k_{m}} \subset \mathbb{R}$ and any $n \in \mathbb{N}$, where $C=c_{r, p}^{m}$ if $0<p<r<2$ or $0<p \leq r=2, C=c_{2,2}^{m}$ if $r=2<p<\infty$ and $C=c_{r, s}^{m}$ if $r<2$ and $r \leq p$, for any $0<s<r$.

Proof. We begin with the cases $0<p<r<2$ and $0<p \leq r=2$. Note that, by (15), we have

$$
\begin{aligned}
(\star) & :=\left(\sum_{k_{1}, \ldots, k_{m}}\left|a_{k_{1}, \ldots, k_{m}}\right|^{r}\right)^{1 / r}=\left(\sum_{k_{1}, \ldots, k_{m-1}}\left(\sum_{k_{m}}\left|a_{k_{1}, \ldots, k_{m}}\right|^{r}\right)^{\frac{1}{r} r}\right)^{1 / r} \\
& =c_{r, p}^{-1}\left(\sum_{k_{1}, \ldots, k_{m-1}}\left(\int_{0}^{1}\left|\sum_{k_{m}} a_{k_{1}, \ldots, k_{m}} w_{k_{m}}\left(t_{m}\right)\right|^{p} d t_{m}\right)^{r / p}\right)^{1 / r} .
\end{aligned}
$$

Then, applying the continuous Minkowski inequality with $r / p \geq 1$ we obtain

$\sum_{k_{m-1}}\left(\int_{0}^{1}\left|\sum_{k_{m}} a_{k_{1}, \ldots, k_{m}} w_{k_{m}}\left(t_{m}\right)\right|^{p} d t_{m}\right)^{r / p} \leq\left(\int_{0}^{1}\left(\sum_{k_{m-1}}\left|\sum_{k_{m}} a_{k_{1}, \ldots, k_{m}} w_{k_{m}}\left(t_{m}\right)\right|^{r}\right)^{p / r} d t_{m}\right)^{r / p}$,

which together with (17) gives

$$
(\star) \leq c_{r, p}^{-1}\left(\sum_{k_{1}, \ldots, k_{m-2}}\left(\int_{0}^{1}\left(\sum_{k_{m-1}}\left|\sum_{k_{m}} a_{k_{1}, \ldots, k_{m}} w_{k_{m}}\left(t_{m}\right)\right|^{r}\right)^{p / r} d t_{m}\right)^{r / p}\right)^{1 / r} .
$$


Using (15) again, we see that

$$
\left(\sum_{k_{m-1}}\left|\sum_{k_{m}} a_{k_{1}, \ldots, k_{m}} w_{k_{m}}\left(t_{m}\right)\right|^{r}\right)^{p / r}=c_{r, p}^{-p} \int_{0}^{1}\left|\sum_{k_{m-1}} \sum_{k_{m}} a_{k_{1}, \ldots, k_{m}} w_{k_{m}}\left(t_{m}\right) w_{k_{m-1}}\left(t_{m-1}\right)\right|^{p} d t_{m-1}
$$

and, in virtue of (18),

$$
(\star) \leq c_{r, p}^{-2}\left(\sum_{k_{1}, \ldots, k_{m-2}}\left(\left.\int_{0}^{1} \int_{0}^{1} \sum_{k_{m-1}, k_{m}} a_{k_{1}, \ldots, k_{m}} w_{k_{m}}\left(t_{m}\right) w_{k_{m-1}}\left(t_{m-1}\right)\right|^{p} d t_{m-1} d t_{m}\right)^{r / p}\right)^{1 / r} .
$$

Repeating this argument, in $(m-1)$-steps we obtain

$$
(\star) \leq c_{r, p}^{-(m-1)}\left(\sum_{k_{1}}\left(\int_{0}^{1} \cdots \int_{0}^{1}\left|\sum_{k_{2}, \ldots, k_{m}} a_{k_{1}, \ldots, k_{m}} w_{k_{m}}\left(t_{m}\right) \cdots w_{k_{2}}\left(t_{2}\right)\right|^{p} d t_{2} \ldots d t_{m}\right)^{r / p}\right)^{1 / r}
$$

and applying Minkowski's inequality (with $r / p \geq 1$ ) and (15) we get

$$
(\star) \leq c_{r, p}^{-m}\left(\int_{0}^{1} \cdots \int_{0}^{1}\left|\sum_{k_{1}, \ldots, k_{m}} a_{k_{1}, \ldots, k_{m}} w_{k_{1}}\left(t_{1}\right) \cdots w_{k_{m}}\left(t_{m}\right)\right|^{p} d t_{1} \cdots d t_{m}\right)^{1 / p},
$$

which proves the statement.

The remaining cases, where $p$ is greater than or equal to $r$, follow choosing $s=r=2$ or $s<r<2$ and applying the previous cases to get

$$
\begin{aligned}
c_{r, s}^{m}\left(\sum_{k_{1}, \ldots, k_{m}}\left|a_{k_{1}, \ldots, k_{m}}\right|^{r}\right)^{1 / r} & \leq\left\|\sum_{k_{1}, \ldots, k_{m}} a_{k_{1}, \ldots, k_{m}} w_{k_{1}} \cdots w_{k_{m}}\right\|_{L^{s}\left([0,1]^{m}\right)} \\
& \leq\left\|\sum_{k_{1}, \ldots, k_{m}} a_{k_{1}, \ldots, k_{m}} w_{k_{1}} \cdots w_{k_{m}}\right\|_{L^{p}\left([0,1]^{m}\right)} .
\end{aligned}
$$

Another useful tool in the proof of the monotonicity in $r$, is the following generalization to the multilinear setting of a result that can be found inside the proof of [17, Thm. 1].

Lemma 2.5. Let $1 \leq q_{1}, \ldots, q_{m}, p \leq \infty$ and $1 \leq r<\infty$. Then

$$
\begin{array}{r}
\left\|\left(\int_{0}^{1} \cdots \int_{0}^{1}\left|\sum_{k_{1}, \ldots, k_{m}=1}^{n} T\left(f_{k_{1}}^{1}, \ldots, f_{k_{m}}^{m}\right) g_{k_{1}}^{1}\left(t_{1}\right) \cdots g_{k_{m}}^{m}\left(t_{m}\right)\right|^{r} d t_{1} \ldots d t_{m}\right)^{1 / r}\right\|_{L^{p}(\nu)} \\
\leq k_{\vec{q}, p}(r)\|T\| \prod_{i=1}^{m}\left\|\left(\int_{0}^{1}\left|\sum_{k_{i}=1}^{n} f_{k_{i}}^{i} g_{k_{i}}^{i}\left(t_{i}\right)\right|^{r} d t_{i}\right)^{1 / r}\right\|_{L^{q_{i}}\left(\mu_{i}\right)}
\end{array}
$$


for each bounded multilinear operator $T: L^{q_{1}}\left(\mu_{1}\right) \times \cdots \times L^{q_{m}}\left(\mu_{m}\right) \rightarrow L^{p}(\nu)$, functions $\left\{f_{k_{i}}^{i}\right\}_{k_{i}=1}^{n} \subset$ $L^{q_{i}}\left(\mu_{i}\right),\left\{g_{k_{i}}^{i}\right\}_{k_{i}=1}^{n} \subset L^{r}[0,1](i=1, \ldots, m)$ and $n \in \mathbb{N}$.

Proof. Fix $T: L^{q_{1}}\left(\mu_{1}\right) \times \cdots \times L^{q_{m}}\left(\mu_{m}\right) \rightarrow L^{p}(\nu), n \in \mathbb{N}$ and $\left\{f_{k_{i}}^{i}\right\}_{k_{i}=1}^{n} \subset L^{q_{i}}\left(\mu_{i}\right),\left\{g_{k_{i}}^{i}\right\}_{k_{i}=1}^{n} \subset$ $L^{r}[0,1]$. Along the proof, we will write $\|\cdot\|_{r}$ instead of $\|\cdot\|_{L^{r}[0,1]}$ or $\|\cdot\|_{L^{r}[0,1]^{m}}$, depending on the context. Let $\varepsilon>0$ and, for each $1 \leq i \leq m$, consider a sequence of simple functions $\left\{s_{k_{i}}^{i}\right\}_{k_{i}=1}^{n}$ such that $\left\|g_{k_{i}}^{i}-s_{k_{i}}^{i}\right\|_{r}<\varepsilon, k_{i}=1, \ldots, n$. On the one hand, reasoning as in [17, Thm. 1], we obtain

$$
\|\| \sum_{k_{i}=1}^{n} f_{k_{i}}^{i} s_{k_{i}}^{i}\|\|_{r}\left\|_{q_{i}} \leq\right\|\left\|\sum_{k_{i}=1}^{n} f_{k_{i}}^{i} g_{k_{i}}^{i}\right\|_{r}\left\|_{q_{i}}+\varepsilon\right\| \sum_{k_{i}=1}^{n}\left|f_{k_{i}}^{i}\right|\left\|_{q_{i}} \leq\right\|\left\|\sum_{k_{i}=1}^{n} f_{k_{i}}^{i} g_{k_{i}}^{i}\right\|_{r}\left\|_{q_{i}}+\varepsilon \sum_{k_{i}=1}^{n}\right\| f_{k_{i}}^{i} \|_{q_{i}} .
$$

On the other hand, $\left\|g_{k_{1}}^{1} \cdots g_{k_{m}}^{m}-s_{k_{1}}^{1} \cdots s_{k_{m}}^{m}\right\|_{r}<\gamma(\varepsilon)$ for some $\gamma(\varepsilon) \underset{\varepsilon \rightarrow 0}{\longrightarrow} 0$ (just add and subtract the terms $s_{k_{1}}^{1} g_{k_{2}}^{2} \cdots g_{k_{m}}^{m}, s_{k_{1}}^{1} s_{k_{2}}^{2} \cdots g_{k_{m}}^{m}, \ldots, s_{k_{1}}^{1} \cdots s_{k_{m-1}}^{m-1} g_{k_{m}}^{m}$ and apply triangle inequality). Then, we have

$$
\begin{aligned}
& \left|\left\|\sum_{k_{1}, \ldots, k_{m}=1}^{n} T\left(f_{k_{1}}^{1}, \ldots, f_{k_{m}}^{m}\right)(\omega) g_{k_{1}}^{1} \cdots g_{k_{m}}^{m}\right\|_{r}-\left\|\sum_{k_{1}, \ldots, k_{m}=1}^{n} T\left(f_{k_{1}}^{1}, \ldots, f_{k_{m}}^{m}\right)(\omega) s_{k_{1}}^{1} \cdots s_{k_{m}}^{m}\right\|_{r}\right| \\
\leq & \sum_{k_{1}, \ldots, k_{m}=1}^{n}\left|T\left(f_{k_{1}}^{1}, \ldots, f_{k_{m}}^{m}\right)(\omega)\right|\left\|g_{k_{1}}^{1} \cdots g_{k_{m}}^{m}-s_{k_{1}}^{1} \cdots s_{k_{m}}^{m}\right\|_{r} \leq \gamma(\varepsilon) \sum_{k_{1}, \ldots, k_{m}=1}^{n}\left|T\left(f_{k_{1}}^{1}, \ldots, f_{k_{m}}^{m}\right)(\omega)\right| .
\end{aligned}
$$

Note that $\gamma(\varepsilon)$ is independent of the $k_{i}$ 's; it depends on $\max _{i, k_{i}}\left\|g_{k_{i}}^{i}\right\|_{r}$ but this is not a problem since the functions $\left\{g_{k_{i}}^{i}\right\}_{k_{i}}$ are fixed. Now, the previous inequality together with the monotonicity of the norm $\|\cdot\|_{p}$, gives

$$
\begin{array}{r}
(\dagger):=\left\|\sum_{k_{1}, \ldots, k_{m}=1}^{n} T\left(f_{k_{1}}^{1}, \ldots, f_{k_{m}}^{m}\right) g_{k_{1}}^{1} \cdots g_{k_{m}}^{m}\right\|_{r}\left\|_{p} \leq\right\|\left\|\sum_{k_{1}, \ldots, k_{m}=1}^{n} T\left(f_{k_{1}}^{1}, \ldots, f_{k_{m}}^{m}\right) s_{k_{1}}^{1} \cdots s_{k_{m}}^{m}\right\|_{r} \|_{p} \\
\quad+\gamma(\varepsilon)\left\|\sum_{k_{1}, \ldots, k_{m}=1}^{n}\left|T\left(f_{k_{1}}^{1}, \ldots, f_{k_{m}}^{m}\right)\right|\right\|_{p} .
\end{array}
$$

Then, if we prove that

$$
\|\| \sum_{k_{1}, \ldots, k_{m}=1}^{n} T\left(f_{k_{1}}^{1}, \ldots, f_{k_{m}}^{m}\right) s_{k_{1}}^{1} \cdots s_{k_{m}}^{m}\left\|_{r}\right\|_{p} \leq k_{\vec{q}, p}(r)\|T\| \prod_{i=1}^{m}\|\| \sum_{k_{i}=1}^{n} f_{k_{i}}^{i} s_{k_{i}}^{i}\left\|_{r}\right\|_{q_{i}},
$$


we would have

$$
\begin{aligned}
(\dagger) \leq k_{\vec{q}, p}(r)\|T\| \prod_{i=1}^{m}\|\| \sum_{k_{i}=1}^{n} f_{k_{i}}^{i} s_{k_{i}}^{i}\left\|_{r}\right\|_{q_{i}}+\gamma(\varepsilon)\left\|_{k_{1}, \ldots, k_{m}=1}^{n} \mid T\left(f_{k_{1}}^{1}, \ldots, f_{k_{m}}^{m}\right)\right\| \|_{p} \quad \text { (by (21) and (22) ) } \\
\left.\leq k_{\vec{q}, p}(r)\|T\| \prod_{i=1}^{m}\left(\|\| \sum_{k_{i}=1}^{n} f_{k_{i}}^{i} g_{k_{i}}^{i}\left\|_{r_{r}}\right\|_{q_{i}}+\varepsilon \sum_{k_{i}=1}^{n}\left\|f_{k_{i}}^{i}\right\|_{q_{i}}\right)+\gamma(\varepsilon)\|T\| \sum_{k_{1}, \ldots, k_{m}=1}^{n} \prod_{i=1}^{m}\left\|f_{k_{i}}^{i}\right\|_{q_{i}}, \quad \text { (by (20) }\right)
\end{aligned}
$$

and since $\varepsilon>0$ was chosen arbitrarily, letting $\varepsilon \rightarrow 0$,

$$
(\dagger) \leq k_{\vec{q}, p}(r)\|T\| \prod_{i=1}^{m}\|\| \sum_{k_{i}=1}^{n} f_{k_{i}}^{i} g_{k_{i}}^{i}\|\|_{r} \|_{q_{i}}
$$

which is the desired statement. Then, it only remains to prove (22). Since each $s_{k_{j}}^{j}$ is a simple function, there exist $c_{i_{j}, k_{j}}^{j} \in \mathbb{R}$ and $A_{i_{j}}$ measurable (disjoint) subsets of [0,1] such that $s_{k_{j}}^{j}(\cdot)=\sum_{i_{j}} c_{i_{j}, k_{j}}^{j} \chi_{A_{i_{j}}}(\cdot)$. Now, if we denote by $\lambda\left(A_{i_{j}}\right)$ the measure of $A_{i_{j}}$,

$$
\begin{aligned}
& \|\| \sum_{k_{1}, \ldots, k_{m}=1}^{n} T\left(f_{k_{1}}^{1}, \ldots, f_{k_{m}}^{m}\right) s_{k_{1}}^{1} \ldots s_{k_{m}}^{m}\left\|_{r}\right\|_{p} \\
= & \left(\int\left(\left.\int_{0}^{1} \cdots \int_{0}^{1} \sum_{k_{1}, \ldots, k_{m}=1}^{n} T\left(f_{k_{1}}^{1}, \ldots, f_{k_{m}}^{m}\right)(\omega) s_{k_{1}}^{1}\left(t_{1}\right) \cdots s_{k_{m}}^{m}\left(t_{m}\right)\right|^{r} d t_{1} \cdots d t_{m}\right)^{p / r} d \nu(\omega)\right)^{1 / p} \\
= & \left(\int\left(\sum_{i_{1}, \ldots, i_{m}}\left|\sum_{k_{1}, \ldots, k_{m}=1}^{n} T\left(f_{k_{1}}^{1}, \ldots, f_{k_{m}}^{m}\right)(\omega) c_{i_{1}, k_{1}}^{1} \ldots c_{i_{m}, k_{m}}^{m} \lambda\left(A_{i_{1}}\right)^{1 / r} \cdots \lambda\left(A_{i_{m}}\right)^{1 / r}\right|^{r}\right)^{p / r} d \nu(\omega)\right)^{1 / p} \\
= & \left(\int\left(\sum_{i_{1}, \ldots, i_{m}}\left|T\left(\sum_{k_{1}=1}^{n} c_{i_{1}, k_{1}}^{1} \lambda\left(A_{i_{1}}\right)^{1 / r} f_{k_{1}}^{1}, \ldots, \sum_{k_{m}=1}^{n} c_{i_{m}, k_{m}}^{m} \lambda\left(A_{i_{m}}\right)^{1 / r} f_{k_{m}}^{m}\right)(\omega)\right|^{r}\right)^{p / r} d \nu(\omega)\right)^{1 / p} \\
\leq & k_{\vec{q}, p}(r)\|T\| \prod_{i=1}^{m}\|\| \sum_{k_{i}=1}^{n} f_{k_{i}}^{i} s_{k_{i}}^{i}\|\|_{r} \|_{q_{i}}
\end{aligned}
$$

which proves (22) (once again, we are assuming $1 \leq p<\infty$, the case $p=\infty$ being completely analogous).

Finally, we prove the monotonicity in $r$ stated in Proposition 1.5 (ii).

Proof of monotonicity in $r$. Let $1 \leq r<s \leq 2$. The proof follows by a simple application of Lemma 2.4 and Lemma 2.5 with the sequences $\left\{g_{k_{i}}^{i}\right\}_{k_{i}}=\left\{w_{k_{i}}\right\}_{k_{i}}$ of $s$-stable random variables 
and functions $\left\{f_{k_{i}}^{i}\right\}_{k_{i}=1}^{n} \subset L^{q_{i}}\left(\mu_{i}\right)$. Indeed, we have

$$
\begin{aligned}
& c_{s, r}^{m}\left\|\left(\sum_{k_{1}, \ldots, k_{m}}\left|T\left(f_{k_{1}}^{1}, \ldots, f_{k_{m}}^{m}\right)\right|\right)^{1 / s}\right\|_{L^{p}(\nu)} \\
\leq & \left\|\left(\int_{0}^{1} \ldots \int_{0}^{1}\left|\sum_{k_{1}, \ldots, k_{m}} T\left(f_{k_{1}}^{1}, \ldots, f_{k_{m}}^{m}\right) w_{k_{1}}\left(t_{1}\right) \cdots w_{k_{m}}\left(t_{m}\right)\right|^{r} d t_{1} \ldots d t_{m}\right)^{1 / r}\right\|_{L^{p}(\nu)} \quad \text { (by (16) ) } \\
\leq & \left.k_{\vec{q}, p}(r)\|T\| \prod_{i=1}^{m}\left\|\left(\int_{0}^{1}\left|\sum_{k_{i}=1}^{n} f_{k_{i}}^{i} w_{k_{i}}\left(t_{i}\right)\right|^{r} d t_{i}\right)^{1 / r}\right\|_{L^{q_{i}\left(\mu_{i}\right)}} \quad \text { (by (19) ) }\right) \\
= & k_{\vec{q}, p}(r)\|T\| c_{s, r}^{m} \prod_{i=1}^{m}\left\|\left(\sum_{k_{i}=1}^{n}\left|f_{k_{i}}^{i}\right|^{s}\right)^{1 / s}\right\|_{L^{q_{i}\left(\mu_{i}\right)}} \quad(\text { by (15) })
\end{aligned}
$$

and this proves that $k_{\vec{q}, p}^{(n)}(s) \leq k_{\vec{q}, p}(r)$. Consequently, $k_{\vec{q}, p}(s) \leq k_{\vec{q}, p}(r)$.

It is worth mentioning that the above proof does not assure $k_{\vec{q}, p}^{(n)}(s) \leq k_{\vec{q}, p}^{(n)}(r)$ for each $n \in \mathbb{N}$. The problem arises in Lemma 2.5, where we cannot put $k_{\vec{q}, p}^{(n)}(r)$ instead of $k_{\vec{q}, p}(r)$. This problem was stated in [17, Problem 1] in the linear case.

We will see now a kind of interpolation behavior of $k_{\vec{q}, p}(r)$ as a function of $r$. First, we need the following known result (see, for instance, [3, Sections 5.6, 5.7 and 5.8]).

Let $1 \leq r_{1}, r_{2} \leq \infty$ and $T$ be a multilinear operator which is bounded from $L^{q_{1}}\left(\mu_{1}, \ell^{r_{i}}\right) \times$ $\cdots \times L^{q_{m}}\left(\mu_{m}, \ell^{r_{i}}\right)$ into $L^{p}\left(\nu, \ell^{r_{i}}(\mathbb{N} \times \cdots \times \mathbb{N})\right)$ with norm $M_{i}(i=1,2)$. If $\frac{1}{r}=\frac{1-\theta}{r_{1}}+\frac{\theta}{r_{2}}$ with $0<\theta<1$, then $T: L^{q_{1}}\left(\mu_{1}, \ell^{r}\right) \times \cdots \times L^{q_{m}}\left(\mu_{m}, \ell^{r}\right) \rightarrow L^{p}\left(\nu, \ell^{r}(\mathbb{N} \times \cdots \times \mathbb{N})\right)$ with norm less than or equal to $M_{1}^{1-\theta} M_{2}^{\theta}$. The same is true if we put $\ell_{n}^{r}$ instead of $\ell^{r}$, where $\ell_{n}^{r}(\mathbb{N} \times \cdots \times \mathbb{N})$ is the space of sequences $\left(a_{k_{1}, \ldots, k_{m}}\right)_{k_{1}, \ldots, k_{m}=1}^{n}$ with the norm $r$.

As a consequence, we obtain the mentioned property of $k_{\vec{q}, p}(r)$.

Corollary 2.6. Let $1 \leq p, q_{1}, \ldots, q_{m}, r_{1}, r_{2} \leq \infty$. If $\frac{1}{r}=\frac{1-\theta}{r_{1}}+\frac{\theta}{r_{2}}$ with $0<\theta<1$, then $k_{\vec{q}, p}^{(n)}(r) \leq k_{\vec{q}, p}^{(n)}\left(r_{1}\right)^{1-\theta} k_{\vec{q}, p}^{(n)}\left(r_{2}\right)^{\theta}$. Consequently, if $k_{\vec{q}, p}\left(r_{i}\right)<\infty$ for $i=1,2$, then $k_{\vec{q}, p}(r) \leq$ $k_{\vec{q}, p}\left(r_{1}\right)^{1-\theta} k_{\vec{q}, p}\left(r_{2}\right)^{\theta}<\infty$.

Proof. It is enough to prove that, given $T: L^{q_{1}}\left(\mu_{1}\right) \times \cdots \times L^{q_{m}}\left(\mu_{m}\right) \rightarrow L^{p}(\nu)$ with norm $\|T\| \leq 1$, the $\ell_{n}^{r}$-valued extension $T^{\ell_{n}^{r}}: L^{q_{1}}\left(\mu_{1}, \ell_{n}^{r}\right) \times \cdots \times L^{q_{m}}\left(\mu_{m}, \ell_{n}^{r}\right) \rightarrow L^{p}\left(\nu, \ell_{n}^{r}(\mathbb{N} \times \cdots \times \mathbb{N})\right)$ defined as in (5) has norm less than or equal to $k_{\vec{q}, p}^{(n)}\left(r_{1}\right)^{1-\theta} k_{\vec{q}, p}^{(n)}\left(r_{2}\right)^{\theta}$. For this, simply note 


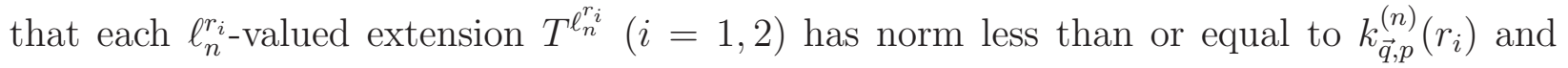
apply the previous interpolation theorem.

2.4. The case $\vec{q}=(1, \ldots, 1)$. To finish this section we prove a generalization to the multilinear setting of a result stated in Theorem $1.2(\mathrm{i})$, which asserts that $k_{1, p}(r)=1$ for all $1 \leq p, r \leq \infty$. Recall that $L^{p}(\mu, X)$ is the space of $p$-integrable $X$-valued functions. When $p=1$, there is a natural (isometric) identification between the projective tensor product $L^{1}(\mu) \tilde{\otimes}_{\pi} X$ and the space $L^{1}(\mu, X)$. Having in mind this isometry, we adopt the tensor notation $g \otimes x(\omega)=g(\omega) x$. We refer readers to [11, 3.3] for the definition of the projective tensor product and a detailed exposition of this topics. For our purposes, we will need the following well-known fact: given $f \in L^{1}(\mu, X)$ (or $L^{1}(\mu) \tilde{\otimes}_{\pi} X$, via the identification) and $\varepsilon>0$ there exist bounded sequences $\left(g_{k}\right)_{k} \subset L^{1}(\mu)$ and $\left(x_{k}\right)_{k} \subset X$ such that the series $\sum_{k=1}^{\infty} g_{k} \otimes x_{k}$ converges to $f$ (in the projective norm) and $\sum_{k=1}^{\infty}\left\|g_{k}\right\|_{L^{1}(\mu)}\left\|x_{k}\right\|_{X}<\|f\|_{L^{1}(\mu, X)}+\varepsilon$. Then, it is clear that

$$
\|f\|_{L^{1}(\mu, X)}=\inf \left\{\sum_{k}\left\|g_{k}\right\|_{L^{1}(\mu)}\left\|x_{k}\right\|_{X}\right\},
$$

where the infimum is taken over all the representations $f=\sum_{k=1}^{\infty} g_{k} \otimes x_{k}$ with $g_{k} \in L^{1}(\mu)$ and $x_{k} \in X$.

Proposition 2.7. If $\vec{q}=(1, \ldots, 1)$, then $k_{\vec{q}, p}(r)=1$ for all $1 \leq p, r \leq \infty$.

Proof. We suppose $1 \leq p, r<\infty$ (the proof is the same in the remaining cases). Let $T: L^{1}\left(\mu_{1}\right) \times \cdots \times L^{1}\left(\mu_{m}\right) \rightarrow L^{p}(\nu)$ and functions $\left\{f_{k_{i}}^{i}\right\}_{k_{i}=1}^{n} \in L^{1}\left(\mu_{i}\right), i=1, \ldots, m$. Consider $h_{i} \in L^{1}\left(\mu_{i}, \ell^{r}\right)$ defined by $h_{i}=\sum_{k_{i}=1}^{n} f_{k_{i}}^{i} \otimes e_{k_{i}}$ and note that

$$
\left\|h_{i}\right\|_{L^{1}\left(\mu_{i}, \ell^{r}\right)}=\int\left\|\sum_{k_{i}=1}^{n} f_{k_{i}}^{i}(\omega) e_{k_{i}}\right\|_{\ell^{r}} d \mu_{i}(\omega)=\left\|\left(\sum_{k_{i}=1}^{n}\left|f_{k_{i}}^{i}\right|^{r}\right)^{1 / r}\right\|_{L^{1}\left(\mu_{i}\right)} .
$$

By (23), given $\varepsilon>0$ we can take (for each $1 \leq i \leq m$ ) a representation $h_{i}=\sum_{l_{i}} g_{l_{i}}^{i} \otimes x_{l_{i}}^{i}$ such that

$$
\sum_{l_{i}}\left\|g_{l_{i}}^{i}\right\|_{L^{1}\left(\mu_{i}\right)}\left\|x_{l_{i}}^{i}\right\|_{\ell^{r}}<\left\|h_{i}\right\|_{L^{1}\left(\mu_{i}, \ell^{r}\right)}+\varepsilon
$$

Now, consider $T^{\ell^{r}}: L^{1}\left(\mu_{1}, \ell^{r}\right) \times \cdots \times L^{1}\left(\mu_{m}, \ell^{r}\right) \rightarrow L^{p}\left(\nu, \ell^{r}(\mathbb{N} \times \cdots \times \mathbb{N})\right)$ the $m$-linear operator defined by $T^{\ell^{r}}\left(f^{1} \otimes x^{1}, \ldots, f^{m} \otimes x^{m}\right)(\cdot)=T\left(f^{1}, \ldots, f^{m}\right)(\cdot) x^{1} \otimes \cdots \otimes x^{m}$, where $x^{1} \otimes \cdots \otimes x^{m}$ 
stands for $\left(x^{1}\left(i_{1}\right) \cdots x^{m}\left(i_{m}\right)\right)_{i_{1}, \ldots, i_{m}=1}^{\infty} \in \ell^{r}(\mathbb{N} \times \cdots \times \mathbb{N})$. On the one hand we have that $T^{\ell^{r}}\left(h_{1}, \ldots, h_{m}\right)(\cdot)=\sum_{k_{1}, \ldots, k_{m}=1}^{n} T\left(f_{k_{1}}^{1}, \ldots, f_{k_{m}}^{m}\right)(\cdot) e_{k_{1}} \otimes \cdots \otimes e_{k_{m}}$ and, hence,

$$
\begin{aligned}
& \left\|T^{\ell^{r}}\left(h_{1}, \ldots, h_{m}\right)\right\|_{L^{p}\left(\nu, \ell^{r}(\mathbb{N} \times \cdots \times \mathbb{N})\right)}=\left(\int\left\|\sum_{k_{1}, \ldots, k_{m}=1}^{n} T\left(f_{k_{1}}^{1}, \ldots, f_{k_{m}}^{m}\right)(\omega) e_{k_{1}} \otimes \cdots \otimes e_{k_{m}}\right\|_{\ell^{r}}^{p} d \nu(\omega)\right)^{1 / p} \\
& =\left(\int\left(\sum_{k_{1}, \ldots, k_{m}=1}^{n}\left|T\left(f_{k_{1}}^{1}, \ldots, f_{k_{m}}^{m}\right)(\omega)\right|^{r}\right)^{p / r} d \nu(\omega)\right)^{1 / p}
\end{aligned}
$$

On the other hand $T^{\ell^{r}}\left(h_{1}, \ldots, h_{m}\right)(\cdot)=\sum_{l_{1}, \ldots, l_{m}} T\left(g_{l_{1}}^{1}, \ldots, g_{l_{m}}^{m}\right)(\cdot) x_{l_{1}}^{1} \otimes \cdots \otimes x_{l_{m}}^{m}$, from where

$$
\begin{aligned}
& \left\|T^{\ell^{r}}\left(h_{1}, \ldots, h_{m}\right)\right\|_{L^{p}\left(\nu, \ell^{r}(\mathbb{N} \times \cdots \times \mathbb{N})\right)}=\left\|\sum_{l_{1}, \ldots, l_{m}} T\left(g_{l_{1}}^{1}, \ldots, g_{l_{m}}^{m}\right)(\cdot) x_{l_{1}}^{1} \otimes \cdots \otimes x_{l_{m}}^{m}\right\|_{L^{p}\left(\nu, \ell^{r}(\mathbb{N} \times \cdots \times \mathbb{N})\right)} \\
\leq & \sum_{l_{1}, \ldots, l_{m}}\left\|T\left(g_{l_{1}}^{1}, \ldots, g_{l_{m}}^{m}\right)\right\|_{L^{p}(\nu)}\left\|x_{l_{1}}^{1}\right\|_{\ell^{r}} \cdots\left\|x_{l_{m}}^{m}\right\|_{\ell^{r}} \leq\|T\| \sum_{l_{1}, \ldots, l_{m}}\left(\prod_{i=1}^{m}\left\|g_{l_{i}}^{i}\right\|_{L^{1}\left(\mu_{i}\right)}\right)\left(\prod_{i=1}^{m}\left\|x_{l_{i}}^{i}\right\|_{\ell^{r}}\right) \\
= & \|T\| \prod_{i=1}^{m}\left(\sum_{l_{i}}\left\|g_{l_{i}}^{i}\right\|_{L^{1}\left(\mu_{i}\right)}\left\|x_{l_{i}}^{i}\right\|_{\ell^{r}}\right) \leq\|T\| \prod_{i=1}^{m}\left(\left\|\left(\sum_{k_{i}=1}^{n}\left|f_{k_{i}}^{i}\right|^{r}\right)^{1 / r}\right\|_{L^{1}\left(\mu_{i}\right)}+\varepsilon\right),
\end{aligned}
$$

the last inequality due to (24) and (25). Putting together (26) and the inequality above we obtain

$$
\left(\int\left(\sum_{k_{1}, \ldots, k_{m}=1}^{n}\left|T\left(f_{k_{1}}^{1}, \ldots, f_{k_{m}}^{m}\right)(\omega)\right|^{r}\right)^{p / r} d \nu(\omega)\right)^{1 / p} \leq\|T\| \prod_{i=1}^{m}\left(\left\|\left(\sum_{k_{i}=1}^{n}\left|f_{k_{i}}^{i}\right|^{r}\right)^{1 / r}\right\|_{L^{1}\left(\mu_{i}\right)}+\varepsilon\right)
$$

and since $\varepsilon>0$ was arbitrary we deduce that $k_{(1, \ldots, 1), p}^{(n)}(r)=1$ for all $n \in \mathbb{N}$.

\section{The TRIPLES $(\vec{q}, p, r)$ SATISFYING $k_{\vec{q}, p}(r)<\infty$}

3.1. The case $1 \leq q_{1}, \ldots, q_{m} \leq 2, p<\infty$. In this section we see that, in the particular case $1 \leq q_{1}, \ldots, q_{m} \leq 2$ and $1 \leq p<\infty$, we can determine the triples $(p, \vec{q}, r)$ satisfying the Marcinkiewicz-Zygmund inequality and the exact values of the constants $k_{\vec{q}, p}(r)$. We follow ideas of [17] (see Theorems 2 and 3 in there) to obtain upper estimates for the constants $k_{\vec{q}, p}(r)$ and then use Proposition 1.4 for the lower bounds. The exact value of $k_{\vec{q}, p}(r)$ is then determined by the exact values of the constants $k_{q_{i}, p}(r)$ obtained in [12].

Theorem 3.1. Let $1 \leq q_{1}, \ldots, q_{m} \leq 2,1 \leq p<\infty$ and denote $\boldsymbol{q}=\max \left\{q_{1}, \ldots, q_{m}\right\}$. Then $k_{\vec{q}, p}(r)<\infty$ if and only if one of the following conditions is satisfied: 
(i) $r=2$;

(ii) $\boldsymbol{q}=1$ and $1 \leq r \leq \infty$;

(iii) $\boldsymbol{q}<r \leq \max \{p, 2\}$;

(iv) $\boldsymbol{q}=r \leq p$.

Moreover, in all these cases we have $k_{\vec{q}, p}(r)=k_{q_{1}, p}(r) \cdots k_{q_{m}, p}(r)$.

It can be easily deduced from the preceding theorem and Theorem 1.2 that, in the case $1 \leq q_{1}, \ldots, q_{m} \leq 2$ and $1 \leq p<\infty$, we have $k_{\vec{q}, p}(r)<\infty$ if and only if $k_{q_{i}, p}(r)<\infty$ for all $1 \leq i \leq m$. Then, in virtue of the monotonicity of $k_{q_{i}, p}(r)$ as a function of $q_{i}$ (see Theorem 1.1), it follows that $k_{\vec{q}, p}(r)<\infty$ if and only if $k_{\boldsymbol{q}, p}(r)<\infty$. Note that, as pointed out in the Introduction (see Proposition 1.7 and the paragraph above), this equivalence does not hold in general, as we will see in Section 4.

Proof of Theorem 3.1. Suppose first that $k_{\vec{q}, p}(r)<\infty$ and (ii) is not satisfied, and let us see that either (i), (iii) or (iv) is satisfied. By Proposition 1.4 we have $k_{q_{i}, p}(r)<\infty$ for all $1 \leq i \leq m$ and, in particular, $k_{\boldsymbol{q}, p}(r)<\infty$. If $\boldsymbol{q} \leq p$, we have $1 \leq \boldsymbol{q} \leq p<\infty$ and $k_{\boldsymbol{q}, p}(r)<\infty$ and, by Theorem [1.2, it follows that $\boldsymbol{q} \leq r \leq \max \{p, 2\}$. If $\boldsymbol{q}>p$, then we have $1 \leq p<\boldsymbol{q} \leq 2$ and $k_{\boldsymbol{q}, p}(r)<\infty$ and by Theorem 1.2 it follows that $r=2$ or $\boldsymbol{q}<r \leq$ $2=\max \{p, 2\}$. We deduce from the cases above that either $r=2$ or $\boldsymbol{q}<r \leq \max \{p, 2\}$ or $\boldsymbol{q}=r$ and $\boldsymbol{q} \leq p$. For the converse, assume the following facts.

(a) If $1 \leq p, \boldsymbol{q}<r<2$ or $1 \leq p, \boldsymbol{q} \leq r=2$, then $k_{\vec{q}, p}(r)=k_{q_{1}, p}(r) \cdots k_{q_{m}, p}(r)<\infty$.

(b) If $\boldsymbol{q} \leq r \leq p$, then $k_{\vec{q}, p}(r)=1=k_{q_{1}, p}(r) \cdots k_{q_{m}, p}(r)$.

If (i) holds then, whether $p<2$ or $p \geq 2, k_{\vec{q}, p}(r)=k_{q_{1}, p}(r) \cdots k_{q_{m}, p}(r)<\infty$ in virtue of (a) and (b). If (ii) holds then by Proposition 2.7 we have $k_{\vec{q}, p}(r)=1=k_{q_{1}, p}(r) \cdots k_{q_{m}, p}(r)$. Suppose we are under the hypothesis of (iii). If $p<r$ then $\boldsymbol{q}<r \leq \max \{p, 2\}=2$ and hence $k_{\vec{q}, p}(r)=k_{q_{1}, p}(r) \cdots k_{q_{m}, p}(r)<\infty$ by (a), while if $p \geq r$ then $\boldsymbol{q}<r \leq p$ and, consequently, $k_{\vec{q}, p}(r)=1=k_{q_{1}, p}(r) \cdots k_{q_{m}, p}(r)$ by (b). Finally, if (iv) holds then $k_{\vec{q}, p}(r)=$ $1=k_{q_{1}, p}(r) \cdots k_{q_{m}, p}(r)$ by (b). Then, it only remains to prove (a) since the assertion in (b) was already stated in Remark 2.3. Fix $T: L^{q_{1}}\left(\mu_{1}\right) \times \cdots \times L^{q_{m}}\left(\mu_{m}\right) \rightarrow L^{p}(\nu)$ and functions 
$\left\{f_{k_{i}}^{i}\right\}_{k_{i}=1}^{n} \subset L^{q_{i}}\left(\mu_{i}\right), 1 \leq i \leq m$. By Lemma 2.4 and the $m$-linearity of $T$ we have

$$
\begin{aligned}
& (\star)=c_{r, p}^{m p} \int\left(\sum_{k_{1}, \ldots, k_{m}=1}^{n}\left|T\left(f_{k_{1}}^{1}, \ldots, f_{k_{m}}^{m}\right)(\omega)\right|^{r}\right)^{p / r} d \nu(\omega) \\
\leq & \iint_{0}^{1} \cdots \int_{0}^{1}\left|\sum_{k_{1}, \ldots, k_{m}=1}^{n} T\left(f_{k_{1}}^{1}, \ldots, f_{k_{m}}^{m}\right)(\omega) w_{k_{1}}\left(t_{1}\right) \cdots w_{k_{m}}\left(t_{m}\right)\right|^{p} d t_{1} \cdots d t_{m} d \nu(\omega) \\
= & \iint_{0}^{1} \cdots \int_{0}^{1}\left|T\left(\sum_{k_{1}=1}^{n} f_{k_{1}}^{1} w_{k_{1}}\left(t_{1}\right), \ldots, \sum_{k_{m}=1}^{n} f_{k_{m}}^{m} w_{k_{m}}\left(t_{m}\right)\right)(\omega)\right|^{p} d t_{1} \cdots d t_{m} d \nu(\omega) .
\end{aligned}
$$

Applying Fubini and the boundedness of $T$ we obtain,

$$
\begin{aligned}
(\star) & \leq \int_{0}^{1} \cdots \int_{0}^{1}\left\|T\left(\sum_{k_{1}=1}^{n} f_{k_{1}}^{1} w_{k_{1}}\left(t_{1}\right), \cdots, \sum_{k_{m}=1}^{n} f_{k_{m}}^{m} w_{k_{m}}\left(t_{m}\right)\right)\right\|_{L^{p}(\nu)}^{p} d t_{1} \cdots d t_{m} \\
& =\|T\|^{p} \prod_{i=1}^{m} \int_{0}^{1}\left\|\sum_{k_{i}=1}^{n} f_{k_{i}}^{i} w_{k_{i}}\left(t_{i}\right)\right\|_{L^{q_{i}\left(\mu_{i}\right)}}^{p} d t_{i} .
\end{aligned}
$$

Then, it will be suffice to obtain upper bounds for each $\int_{0}^{1}\left\|\sum_{k_{i}=1}^{n} f_{k_{i}}^{i} w_{k_{i}}\left(t_{i}\right)\right\|_{L^{q_{i}\left(\mu_{i}\right)}}^{p} d t_{i}$. On the one hand, for those $q_{i}<p$ we have

$$
\begin{aligned}
& \int_{0}^{1}\left\|\sum_{k_{i}=1}^{n} f_{k_{i}}^{i} w_{k_{i}}\left(t_{i}\right)\right\|_{L^{q_{i}\left(\mu_{i}\right)}}^{p} d t_{i}=\left[\left(\int_{0}^{1}\left(\int\left|\sum_{k_{i}=1}^{n} f_{k_{i}}^{i}(\omega) w_{k_{i}}\left(t_{i}\right)\right|^{q_{i}} d \mu_{i}(\omega)\right)^{p / q_{i}} d t_{i}\right)^{q_{i} / p}\right]^{p / q_{i}} \\
\leq & {\left.\left[\int\left(\int_{0}^{1}\left|\sum_{k_{i}=1}^{n} f_{k_{i}}^{i}(\omega) w_{k_{i}}\left(t_{i}\right)\right|^{p} d t_{i}\right)^{q_{i} / p} d \mu_{i}(\omega)\right]^{1 / p} \text { (by Minkowski with } p / q_{i}>1\right) } \\
= & c_{r, p}^{p}\left\|\left(\sum_{k_{i}=1}^{n}\left|f_{k_{i}}^{i}\right|^{r}\right)^{1 / r}\right\|_{L^{q_{i}\left(\mu_{i}\right)}}^{p} \quad(\text { by (15) }) .
\end{aligned}
$$

On the other hand, if $p \leq q_{i}$

$$
\begin{aligned}
\int_{0}^{1}\left\|\sum_{k_{i}=1}^{n} f_{k_{i}}^{i} w_{k_{i}}\left(t_{i}\right)\right\|_{L^{q_{i}\left(\mu_{i}\right)}}^{p} d t_{i} & \left.\leq\left(\int_{0}^{1}\left\|\sum_{k_{i}=1}^{n} f_{k_{i}}^{i} w_{k_{i}}\left(t_{i}\right)\right\|_{L^{q_{i}\left(\mu_{i}\right)}}^{q_{i}} d t_{i}\right)^{p / q_{i}} \quad \text { (by Hölder with } q_{i} / p \geq 1\right) \\
& =\left(\iint_{0}^{1}\left|\sum_{k_{i}=1}^{n} f_{k_{i}}^{i}(\omega) w_{k_{i}}\left(t_{i}\right)\right|^{q_{i}} d t_{i} d \mu_{i}(\omega)\right)^{p / q_{i}} \quad \text { (by Fubini) } \\
& =c_{r, q_{i}}^{p}\left\|\left(\sum_{k_{i}=1}^{n}\left|f_{k_{i}}^{i}\right|^{r}\right)^{1 / r}\right\|_{L^{q_{i}\left(\mu_{i}\right)}}^{p} \quad \text { (by (15)) ). }
\end{aligned}
$$


These inequalities together with (27) give

$$
\left\|\left(\sum_{k_{1}, \ldots, k_{m}=1}^{n}\left|T\left(f_{k_{1}}^{1}, \ldots, f_{k_{m}}^{m}\right)\right|^{r}\right)^{1 / r}\right\|_{L^{p}(\nu)} \leq \frac{c_{r, q_{j_{1}}} \cdots c_{r, q_{j m_{2}}}}{c_{r, p}^{m_{2}}}\|T\| \prod_{i=1}^{m}\left\|\left(\sum_{k_{i}=1}^{n}\left|f_{k_{i}}^{i}\right|^{r}\right)^{1 / r}\right\|_{L^{q_{i}}\left(\mu_{i}\right)},
$$

where $q_{j_{1}}, \ldots, q_{j_{m_{2}}}$ are those $q_{i} \geq p$. Noting that $k_{q_{i}, p}(r)=1$ if $q_{i}<p$ and $k_{q_{i}, p}(r)=\frac{c_{r, q_{i}}}{c_{r, p}}$ if $q_{i} \geq p$ (see Theorem 1.2), the last inequality gives $k_{\vec{q}, p}(r) \leq k_{q_{1}, p}(r) \cdots k_{q_{m}, p}(r)<\infty$. The equality holds as a consequence of Proposition 1.4 and this proves (a).

Note that in the proof of the statement (a) when $r<2$, the hypothesis $p, \boldsymbol{q}<r$ is necessary in order to apply (15). When $r=2$, there is no need of this hypothesis to see, with the same proof, that $k_{\vec{q}, p}(2)<\infty$ (we will point out this fact in Proposition 5.3 below). In this case, we need the assumption $p, \boldsymbol{q} \leq 2$ to ensure $k_{\vec{q}, p}(2)=k_{q_{1}, p}(2) \cdots k_{q_{m}, p}(2)$.

3.2. Proof of the main theorem. We prove now Theorem 1.3, which determines the set of those triples $(p, \vec{q}, r)$ satisfying $k_{\vec{q}, p}(r)<\infty$, with the exception of the cases $p=\infty$ (partially discussed in Section (4) and $2 \leq p<\max \left\{q_{1}, \ldots, q_{m}\right\}$. At this point, the results stated in the theorem are simple consequences of those obtained in the previous sections.

Proof of Theorem 1.3. We begin by proving (i). Items (ia) and (ic) are consequences of Theorem 3.1, then we only need to prove (ib). If $k_{\vec{q}, p}(r)<\infty$ then $k_{\boldsymbol{q}, p}(r)<\infty$ (by Proposition 1.4) and by Theorem 1.2(ii) it follows that $2 \leq r \leq p$. For the converse, take $2 \leq r \leq p$. If $\boldsymbol{q} \leq r \leq p$ then $k_{\vec{q}, p}(r)=1$ by Remark 2.3, while if $2 \leq r \leq \boldsymbol{q}$ then $k_{\vec{q}, p}(r)<\infty$ by Corollary 2.6, since $k_{\vec{q}, p}(2)<\infty$ (this is the particular case of the Marcinkiewicz-Zygmund inequalities proved in [5, 18]) and $k_{\vec{q}, p}(\boldsymbol{q})=1<\infty$.

Now we prove (ii). Item (iia) follows from Theorem 3.1. For (iib) (resp. (iic)) note that $k_{\vec{q}, p}(r)<\infty$ implies $k_{\boldsymbol{q}, p}(r)<\infty$ and, by Theorem 1.2(iii), this gives $2 \leq r<p$ (resp. $r=2$ ). The converse in (iic) and the sufficient condition in (iib) are again consequences of the multilinear Marcinkiewicz-Zygmund inequality for $r=2$ obtained in [5, 18].

\section{Proof of Proposition 1.7}

The key tool in this section is a variant of a Kahane-Salem-Zygmund inequality. The idea is to construct a multilinear operator from $\ell_{n}^{q_{1}} \times \cdots \times \ell_{n}^{q_{m}}$ into $\ell_{n}^{\infty}$ with relatively small supremum norm but for which $\left(\sum_{i_{1}, \ldots, i_{m}=1}^{n}\left|T\left(e_{i_{1}}, \ldots, e_{i_{m}}\right)\right|^{r}\right)^{1 / r}$ has a relatively large $\ell^{\infty}$-norm. This, 
together with an appropriate choice of the indices $q_{1}, \ldots, q_{m}, r$, will force $\lim _{n} k_{\vec{q}, \infty}^{(n)}(r)=\infty$ as desired. In [4, Thm. 4], Boas showed that there exists an $(m+1)$-linear map $T: \ell_{n}^{2} \times \cdots \times \ell_{n}^{2} \rightarrow$ $\mathbb{R}$ of the form

$$
T\left(z^{(1)}, \ldots, z^{(m+1)}\right)=\sum_{j_{1}, \ldots, j_{m+1}=1}^{n} \varepsilon_{j_{1}, \ldots, j_{m+1}} z_{j_{1}}^{(1)} \cdots z_{j_{m+1}}^{(m+1)}
$$

where $\varepsilon_{j_{1}, \ldots, j_{m+1}}= \pm 1$, such that $\|T\|_{\ell_{n}^{2} \times \cdots \times \ell_{n}^{2} \rightarrow \mathbb{R}} \prec n^{1 / 2}$ (here, $\prec$ means that there exist a constant $C_{m}>0$, depending only on $m$, such that $\left.\|T\|_{\ell_{n}^{2} \times \cdots \times \ell_{n}^{2} \rightarrow \mathbb{R}} \leq C_{m} n^{1 / 2}\right)$. The restriction of this operator to $\ell_{n}^{1} \times \cdots \times \ell_{n}^{1}$ has norm one and hence, by an interpolation argument, if $1<q<2$ (note that $\frac{1}{q}=\frac{1-\theta}{1}+\frac{\theta}{2}$ with $\theta=\frac{2}{q^{\prime}}$ ) then $T: \ell_{n}^{q} \times \cdots \times \ell_{n}^{q} \rightarrow \mathbb{R}$ has norm $\|T\|_{\ell_{n}^{q} \times \cdots \times \ell_{n}^{q} \rightarrow \mathbb{R}} \prec 1^{1-\theta} n^{\frac{\theta}{2}}=n^{\frac{1}{q^{\prime}}}$. It is clear then that, if $1 \leq q_{1}, \ldots, q_{m+1} \leq 2$ and $\boldsymbol{q}=$ $\max \left\{q_{1}, \ldots, q_{m+1}\right\}, T: \ell_{n}^{q_{1}} \times \cdots \times \ell_{n}^{q_{m+1}} \rightarrow \mathbb{R}$ has norm $\|T\|_{\ell_{n}^{q_{1}} \times \cdots \times \ell_{n}^{q_{m+1}} \rightarrow \mathbb{R}} \leq\|T\|_{\ell_{n}^{q} \times \cdots \times \ell_{n}^{q} \rightarrow \mathbb{R}} \prec$ $n^{\frac{1}{q^{\prime}}}$. If, instead, $1 \leq q_{1}, \ldots, q_{m+1} \leq \infty$ with $q_{i}>2$ for at least one $1 \leq i \leq m+1$, we can take $T: \ell_{n}^{2} \times \cdots \times \ell_{n}^{2} \rightarrow \mathbb{R}$ as in (28) and compose it with the identities $i d_{q_{i}, 2}^{n}: \ell_{n}^{q_{i}} \rightarrow \ell_{n}^{2}$.

$$
\begin{aligned}
\left\|T \circ\left(i d_{q_{1}, 2}^{n}, \ldots, i d_{q_{m+1}, 2}^{n}\right)\right\|_{\ell_{n}^{q_{1}} \times \cdots \times \ell_{n}^{q_{m+1}} \rightarrow \mathbb{R}} & \leq\|T\|_{\ell_{n}^{2} \times \cdots \times \ell_{n}^{2} \rightarrow \mathbb{R}}\left\|i d_{q_{1}, 2}^{n}\right\| \cdots\left\|i d_{q_{m+1}, 2}^{n}\right\| \\
& \prec n^{\frac{1}{2}}\left\|i d_{q_{1}, 2}^{n}\right\| \cdots\left\|i d_{q_{m+1}, 2}^{n}\right\| .
\end{aligned}
$$

Note that $i d_{q_{i}, 2}^{n}$ has norm one if $q_{i} \leq 2$ and norm $n^{\frac{1}{2}-\frac{1}{q_{i}}}$ if $q_{i}>2$. As a consequence of the previous observations and the isometric correspondence between $m$-linear maps $\ell_{n}^{q_{1}} \times \cdots \times$ $\ell_{n}^{q_{m}} \rightarrow \ell_{n}^{q_{m+1}^{\prime}}$ and $(m+1)$-linear maps $\ell_{n}^{q_{1}} \times \cdots \times \ell_{n}^{q_{m+1}} \rightarrow \mathbb{R}$, we obtain the following lemma.

Lemma 4.1. Let $m, n \in \mathbb{N}, 1 \leq q_{1}, \ldots, q_{m}, p \leq \infty$ and $\boldsymbol{q}=\max \left\{q_{1}, \ldots, q_{m}\right\}$. Then there exists an m-linear map $T: \ell_{n}^{q_{1}} \times \cdots \times \ell_{n}^{q_{m}} \rightarrow \ell_{n}^{p}$ of the form

$$
T\left(z^{(1)}, \ldots, z^{(m)}\right)=\sum_{j_{m+1}=1}^{n} \sum_{j_{1}, \ldots, j_{m}=1}^{n} \varepsilon_{j_{1}, \ldots, j_{m+1}} z_{j_{1}}^{(1)} \cdots z_{j_{m}}^{(m)} e_{j_{m+1}}
$$

where $\varepsilon_{j_{1}, \ldots, j_{m+1}}= \pm 1$, such that

$$
\|T\| \prec\left\{\begin{array}{cl}
n^{\frac{1}{\min \left\{\boldsymbol{q}^{\prime}, p\right\}}} & \text { if } \boldsymbol{q}, p^{\prime} \leq 2, \\
n^{\frac{1}{2}}\left\|i d_{q_{1}, 2}^{n}\right\| \cdots\left\|i d_{q_{m}, 2}^{n}\right\|\left\|i d_{p^{\prime}, 2}^{n}\right\| & \text { otherwise. }
\end{array}\right.
$$

Proof of Proposition 1.7. Our goal is to show that $k_{\vec{q}, \infty}^{(n)}(r) \succ n^{\gamma}$ for some $\gamma>0$ independent of $n$, in which case $k_{\vec{q}, \infty}(r)=\lim _{n} k_{\vec{q}, \infty}^{(n)}(r)=\infty$. In virtue of Lemma 4.1, there is an $m$-linear operator $T: \ell_{n}^{q_{1}} \times \cdots \times \ell_{n}^{q_{m}} \rightarrow \ell_{n}^{\infty}$ as in (29) such that

$$
\|T\| \prec n^{\frac{1}{\max \left(\boldsymbol{q}^{\prime}, 2\right)}+\sum_{q_{i}>2}\left(\frac{1}{2}-\frac{1}{q_{i}}\right)},
$$


where $\sum_{q_{i}>2}\left(\frac{1}{2}-\frac{1}{q_{i}}\right)=0$ if $\boldsymbol{q} \leq 2$. Now, since $\left|T\left(e_{i_{1}}, \ldots, e_{i_{m}}\right)\right|=(1, \ldots, 1)$ for all $1 \leq$ $i_{1}, \ldots, i_{m} \leq n$ we have

$$
\left\|\left(\sum_{i_{1}, \ldots, i_{m}=1}^{n}\left|T\left(e_{i_{1}}, \ldots, e_{i_{m}}\right)\right|^{r}\right)^{1 / r}\right\|_{\ell^{\infty}}=\left\|\left(\left(n^{m}, \ldots, n^{m}\right)\right)^{1 / r}\right\|_{\ell^{\infty}}=n^{\frac{m}{r}} .
$$

Then,

$$
\begin{aligned}
n^{\frac{m}{r}} & =\left\|\left(\sum_{i_{1}, \ldots, i_{m}=1}^{n}\left|T\left(e_{i_{1}}, \ldots, e_{i_{m}}\right)\right|^{r}\right)^{1 / r}\right\|_{\ell^{\infty}} \leq k_{\vec{q}, \infty}^{(n)}(r)\|T\| \prod_{i=1}^{m}\left\|\left(\sum_{j=1}^{n}\left|e_{j}\right|^{r}\right)^{1 / r}\right\|_{\ell_{q_{i}}} \\
& \prec k_{\vec{q}, \infty}^{(n)}(r) n^{\frac{1}{\max \left(\boldsymbol{q}^{\prime}, 2\right)}+\frac{1}{\min \left(q_{1}, 2\right)}+\cdots+\frac{1}{\min \left(q_{m}, 2\right)}} .
\end{aligned}
$$

The statement follows from this inequality.

\section{Applications}

\subsection{Weighted vector-valued estimates for multilinear Calderón-Zygmund oper-}

ators. The study of multilinear Calderón-Zygmund theory finds its origins in the seventies with works of Coifman and Meyer, but a systematic treatment of this topic appears later with works of Grafakos and Torres [19, 20]. Recall the definition of a multilinear CalderónZygmund operator: let $T: S\left(\mathbb{R}^{n}\right) \times \cdots \times S\left(\mathbb{R}^{n}\right) \rightarrow S^{\prime}\left(\mathbb{R}^{n}\right)$ be a multilinear operator initially defined on the $m$-fold product of Schwartz spaces and taking values into the space of tempered distributions; we say that $T$ is an $m$-linear Calderón-Zygmund operator if, for some $1 \leq q_{1}, \ldots, q_{m}<\infty$ and $\frac{1}{m} \leq p<\infty$ satisfying $\frac{1}{p}=\frac{1}{q_{1}}+\cdots+\frac{1}{q_{m}}$, it extends to a bounded multilinear operator from $L^{q_{1}} \times \cdots \times L^{q_{m}}$ to $L^{p}$, and if there exists a function $K$ defined off the diagonal $x=y_{1}=\cdots=y_{m}$ in $\left(\mathbb{R}^{n}\right)^{m+1}$ satisfying the appropriate decay and smoothness conditions (see [19, 20]) and such that

$$
T\left(f^{1}, \ldots, f^{m}\right)(x)=\int_{\mathbb{R}^{n}} \ldots \int_{\mathbb{R}^{n}} K\left(x, y_{1}, \ldots, y_{m}\right) \prod_{i=1}^{m} f^{i}\left(y_{i}\right) d y_{1} \cdots d y_{m}
$$

for all $x \notin \cap_{i=1}^{m} \operatorname{supp} f_{i}$. In [19] it was shown that, if $\frac{1}{p}=\frac{1}{q_{1}}+\cdots+\frac{1}{q_{m}}$ with $1<q_{1}, \ldots, q_{m}<\infty$, then an $m$-linear Calderón-Zygmund operator $T$ maps $L^{q_{1}}\left(\mathbb{R}^{n}\right) \times \cdots \times L^{q_{m}}\left(\mathbb{R}^{n}\right)$ into $L^{p}\left(\mathbb{R}^{n}\right)$. If $1 \leq q_{1}, \ldots, q_{m}<\infty$ and at least one $q_{i}=1$, then $T$ maps $L^{q_{1}}\left(\mathbb{R}^{n}\right) \times \cdots \times L^{q_{m}}\left(\mathbb{R}^{n}\right)$ into $L^{p, \infty}\left(\mathbb{R}^{n}\right)$. Regarding the weighted norm inequalities, the first result was obtained in [20] (see also [25]) where the authors proved that, if $1<q_{1}, \ldots, q_{m}<\infty$ and $w$ is a weight in the Muckenhoupt $A_{q_{0}}$ class for $q_{0}=\min \left\{q_{1}, \ldots, q_{m}\right\}$, an $m$-linear Calderón-Zygmund operator $T$ maps $L^{q_{1}}(w) \times \cdots \times L^{q_{m}}(w)$ into $L^{p}(w)$. The same approach of [20] shows that $T$ maps 
$L^{q_{1}}\left(w_{1}\right) \times \cdots \times L^{q_{m}}\left(w_{m}\right)$ into $L^{p}\left(\nu_{\vec{w}}\right)$, where $\nu_{\vec{w}}=\prod_{i=1}^{m} w_{i}^{p / q_{i}}$ and $w_{i}$ is in $A_{q_{i}}$. As expected, if at least one $q_{i}=1$, then the weak endpoint estimate holds. In [23], the authors developed the right class of multiple weights for $m$-linear Calderón-Zygmund operators. We briefly review the weighted estimate proved in there for multilinear Calderón-Zygmund operators. As usual, let $1 \leq q_{1}, \ldots, q_{m}<\infty$ and $\frac{1}{m} \leq p<\infty$ be such that $\frac{1}{p}=\frac{1}{q_{1}}+\cdots+\frac{1}{q_{m}}$. We say that $\vec{w}=\left(w_{1}, \ldots, w_{m}\right)$ satisfies the multilinear $A_{\vec{q}}$ condition if

$$
\sup _{Q}\left(\frac{1}{|Q|} \int_{Q} \nu_{\vec{w}}\right)^{1 / p} \prod_{i=1}^{m}\left(\frac{1}{|Q|} \int_{Q} w_{i}^{1-q_{i}^{\prime}}\right)^{1 / q_{i}^{\prime}}<\infty
$$

where the supremum is taken over all cubes $Q$ (when $q_{i}=1,\left(\frac{1}{|Q|} \int_{Q} w_{i}^{1-q_{i}^{\prime}}\right)^{1 / q_{i}^{\prime}}$ is understood as $\left.\left(\inf _{Q} w_{i}\right)^{-1}\right)$. Now, if $\vec{w}$ satisfies the $A_{\vec{q}}$ condition and $1<q_{1}, \ldots, q_{m}<\infty$, then an $m$-linear Calderón-Zygmund operator $T$ maps $L^{q_{1}}\left(w_{1}\right) \times \cdots \times L^{q_{m}}\left(w_{m}\right)$ into $L^{p}\left(\nu_{\vec{w}}\right)$. If at least one $q_{i}=1$, then $T$ maps $L^{q_{1}}\left(w_{1}\right) \times \cdots \times L^{q_{m}}\left(w_{m}\right)$ into $L^{p, \infty}\left(\nu_{\vec{w}}\right)$. It is shown that $\prod_{i=1}^{m} A_{q_{i}} \subseteq A_{\vec{q}}$ and that this inclusion is strict; then, the above weighted estimates improve those obtained in [20]. In fact, if $T$ is the $m$-linear Riesz Transform, it was proved in [23] that $A_{\vec{q}}$ is a necessary condition for such weighted estimate of $T$.

5.1.1. Some known estimates and their comparison with Marcinkiewicz-Zygmund inequalities. In the context of vector-valued inequalities for multilinear Calderón-Zygmund operators, the following result was proved in [8, Corollary 3.3] by means of extrapolation techniques. See also [9, Section 6.4] where this type of inequalities are obtained in the more general context of rearrangement invariant quasi-Banach function spaces.

Theorem 5.1 [8]. Let $T$ be a multilinear Calderón-Zygmund operator, $1 \leq q_{1}, \ldots, q_{m}<\infty$, $1<r_{1}, \ldots, r_{m}<\infty$ and $0<p, r<\infty$ such that

$$
\frac{1}{p}=\frac{1}{q_{1}}+\cdots+\frac{1}{q_{m}}, \quad \frac{1}{r}=\frac{1}{r_{1}}+\cdots+\frac{1}{r_{m}} .
$$

If $1<q_{1}, \ldots, q_{m}<\infty$ and $w \in A_{q_{0}}$ for $q_{0}=\min \left\{q_{1}, \ldots, q_{m}\right\}$, then

$$
\left\|\left(\sum_{k}\left|T\left(f_{k}^{1}, \ldots, f_{k}^{m}\right)\right|^{r}\right)^{\frac{1}{r}}\right\|_{L^{p}(w)} \leq C \prod_{i=1}^{m}\left\|\left(\sum_{k}\left|f_{k}^{i}\right|^{r_{i}}\right)^{\frac{1}{r_{i}}}\right\|_{L^{q_{i}}(w)} .
$$

If at least one $q_{i}=1$ and $w \in A_{1}$, then

$$
\left\|\left(\sum_{k}\left|T\left(f_{k}^{1}, \ldots, f_{k}^{m}\right)\right|^{r}\right)^{\frac{1}{r}}\right\|_{L^{p, \infty}(w)} \leq C \prod_{i=1}^{m}\left\|\left(\sum_{k}\left|f_{k}^{i}\right|^{r_{i}}\right)^{\frac{1}{r_{i}}}\right\|_{L^{q_{i}(w)}} .
$$


The estimate (32) was obtained independently in [18] as a particular case of the inequality (34) for $m$-tuples of weights. Also a weaker version of (33) was obtained as a consequence of a multilinear Marcinkiewicz-Zygmund inequality for $r=2$.

Theorem 5.2 [18]. Let $T$ be a multilinear Calderón-Zygmund operator, $1 \leq q_{1}, \ldots, q_{m}<\infty$, $1<r_{1}, \ldots, r_{m}<\infty$ and $0<p, r<\infty$ such that

$$
\frac{1}{p}=\frac{1}{q_{1}}+\cdots+\frac{1}{q_{m}}, \quad \frac{1}{r}=\frac{1}{r_{1}}+\cdots+\frac{1}{r_{m}} .
$$

If $1<q_{1}, \ldots, q_{m}<\infty$ and $\left(w_{1}^{q_{1}}, \ldots, w_{m}^{q_{m}}\right) \in A_{q_{1}} \times \cdots \times A_{q_{m}}$, then

$$
\left\|\left(\sum_{k}\left|T\left(f_{k}^{1}, \ldots, f_{k}^{m}\right)\right|^{r}\right)^{\frac{1}{r}}\right\|_{L^{p}\left(w_{1}^{p} \cdots w_{m}^{p}\right)} \leq C \prod_{i=1}^{m}\left\|\left(\sum_{k}\left|f_{k}^{i}\right|^{r_{i}}\right)^{\frac{1}{r_{i}}}\right\|_{L^{q_{i}}\left(w_{i}^{q_{i}}\right)} .
$$

If at least one $q_{i}=1$ and $w \in A_{1}$, then

$$
\left\|\left(\sum_{k}\left|T\left(f_{k}^{1}, \ldots, f_{k}^{m}\right)\right|^{2}\right)^{\frac{1}{2}}\right\|_{L^{p, \infty}(w)} \leq C \prod_{i=1}^{m}\left\|\left(\sum_{k}\left|f_{k}^{i}\right|^{2}\right)^{\frac{1}{2}}\right\|_{L^{q_{i}(w)}} .
$$

It is worth mentioning that, in the above weighted vector-valued estimates, the multilinear $A_{\vec{q}}$ condition is not considered. We will consider this appropriate class of multiple weights in Corollary 5.4, where vector-valued inequalities for Calderón-Zygmund operators are obtained. Also, as we emphasize below, the vector-valued inequalities that we obtain are significantly different from those stated in (32), (33), (34). In our case, the sum on the left-hand side is replaced by a multi-indexed sum over $k_{1}, \ldots, k_{m}$ and we consider only one power $r$ instead of the powers $1<r_{1}, \ldots, r_{m}<\infty$.

At this point we would like to stress the relation $\frac{1}{r}=\frac{1}{r_{1}}+\cdots+\frac{1}{r_{m}}$ that appears in the hypotheses of the previous theorems which, by the way, shows why the estimate (35) is weaker than (33). When proving estimates like (34), one is interested in the study of the following inequalities:

$$
\left\|\left(\sum_{k}\left|T\left(f_{k}^{1}, \ldots, f_{k}^{m}\right)\right|^{s}\right)^{\frac{1}{s}}\right\|_{L^{p}(\nu)} \leq C \prod_{i=1}^{m}\left\|\left(\sum_{k}\left|f_{k}^{i}\right|^{r_{i}}\right)^{\frac{1}{r_{i}}}\right\|_{L^{q_{i}}\left(\mu_{i}\right)} .
$$

If in the previous inequality we put $f_{k}^{i}=f^{i} \in L^{q_{i}}\left(\mu_{i}\right)$ for $1 \leq k \leq n, 1 \leq i \leq m$ and $f_{k}^{i}=0$ otherwise, we obtain

$$
n^{\frac{1}{s}}\left\|T\left(f^{1}, \ldots, f^{m}\right)\right\|_{p} \leq C \prod_{i=1}^{m} n^{\frac{1}{r_{i}}}\left\|f^{i}\right\|_{L^{q_{i}}\left(\mu_{i}\right)}
$$


and, since $C$ is independent of $n$, this forces $\frac{1}{s} \leq \frac{1}{r_{1}}+\cdots+\frac{1}{r_{m}}$. Thus, the estimate (36) is optimal when $s=r$, where $r$ satisfies $\frac{1}{r}=\frac{1}{r_{1}}+\cdots+\frac{1}{r_{m}}$. In the case of (35) the relation between the powers is not optimal as in (33). In fact, if (33) holds for $r=2$ then $2 \leq r_{i}$ for all $1 \leq i \leq m$ and, since $\ell^{2} \hookrightarrow \ell^{r_{i}}$ with $\|\cdot\|_{\ell^{r_{i}}} \leq\|\cdot\|_{\ell^{2}}$, then

$$
\left\|\left(\sum_{k}\left|f_{k}^{i}\right|^{r_{i}}\right)^{\frac{1}{r_{i}}}\right\|_{L^{q_{i}\left(\mu_{i}\right)}} \leq\left\|\left(\sum_{k}\left|f_{k}^{i}\right|^{2}\right)^{\frac{1}{2}}\right\|_{L^{q_{i}\left(\mu_{i}\right)}},
$$

which yields the (hence, weaker) estimate in (35).

Now, the Marcinkiewicz-Zygmund inequalities we were studying in the previous sections have a significant difference with those in (36). In the former ones the sum

$$
\left(\sum_{k_{1}, \ldots, k_{m}}\left|T\left(f_{k_{1}}^{1}, \ldots, f_{k_{m}}^{m}\right)\right|^{r}\right)^{\frac{1}{r}}
$$

ranges over the indices $k_{1}, \ldots, k_{m}$, while in (36) we sum over the diagonal, hence only one index $k$. This produces another optimal relation between the powers $r, r_{1}, \ldots, r_{m}$. Indeed, suppose we are interested in estimates of the form

$$
\left\|\left(\sum_{k_{1}, \ldots, k_{m}}\left|T\left(f_{k_{1}}^{1}, \ldots, f_{k_{m}}^{m}\right)\right|^{s}\right)^{\frac{1}{s}}\right\|_{L^{p}(\nu)} \leq C \prod_{i=1}^{m}\left\|\left(\sum_{k_{i}}\left|f_{k_{i}}^{i}\right|^{r_{i}}\right)^{\frac{1}{r_{i}}}\right\|_{L^{q_{i}}\left(\mu_{i}\right)} .
$$

If we choose $f_{k_{i}}^{i}=f^{i} \in L^{q_{i}}\left(\mu_{i}\right)$ for $1 \leq k_{i} \leq n, 1 \leq i \leq m$ and $f_{k_{i}}^{i}=0$ otherwise, we have

$$
n^{\frac{m}{s}}\left\|T\left(f^{1}, \ldots, f^{m}\right)\right\|_{p} \leq C \prod_{i=1}^{m} n^{\frac{1}{r_{i}}}\left\|f^{i}\right\|_{L^{q_{i}}\left(\mu_{i}\right)}
$$

from where we get $\frac{m}{s} \leq \frac{1}{r_{1}}+\cdots+\frac{1}{r_{m}}$. Also, if in (38) we take any sequence $\left\{f_{k_{1}}^{1}\right\}_{k_{1}} \subset L^{q_{1}}\left(\mu_{1}\right)$ and, for each $2 \leq i \leq m$, we put $f_{1}^{i}=f^{i} \in L^{q_{i}}\left(\mu_{i}\right)$ and $f_{k_{i}}^{i}=0$ for $k_{i} \geq 2$, then we obtain

$$
\left\|\left(\sum_{k_{1}}\left|\tilde{T}\left(f_{k_{1}}^{1}\right)\right|^{s}\right)^{\frac{1}{s}}\right\|_{L^{p}(\nu)} \leq C\left(\prod_{i=2}^{m}\left\|f^{i}\right\|_{L^{q_{i}}\left(\mu_{i}\right)}\right)\left\|\left(\sum_{k_{1}}\left|f_{k_{1}}^{1}\right|^{r_{1}}\right)^{\frac{1}{r_{1}}}\right\|_{L^{q_{1}}\left(\mu_{1}\right)}
$$

where $\tilde{T}: L^{q_{1}}\left(\mu_{1}\right) \rightarrow L^{p}(\nu)$ is the linear operator $\tilde{T}(\cdot)=T\left(\cdot, f^{2}, \ldots, f^{m}\right)$. In virtue of (37) (when $m=1$ ) this last inequality gives $\frac{1}{s} \leq \frac{1}{r_{1}}$. Analogously, $\frac{1}{s} \leq \frac{1}{r_{i}}$ for all $1 \leq i \leq m$. In sum, when we are dealing with inequality (38) we have the following conditions over the powers $s, r_{1}, \ldots, r_{m}$,

$$
\frac{m}{s} \leq \frac{1}{r_{1}}+\cdots+\frac{1}{r_{m}} \quad \text { and } \quad \frac{1}{s} \leq \frac{1}{r_{i}} \quad \text { for all } 1 \leq i \leq m .
$$


This shows that the inequality (38) is optimal when $s=r_{1}=\cdots=r_{m}=r$, which is just the case treated in the Marcinkiewicz-Zygmund inequalities.

Camil Muscalu pointed out to us that, in the unweighted case, Marcinkiewicz-Zygmund estimates for multilinear Calderón-Zygmund operators can be deduced from more general multiple vector-valued inequalities obtained in his recent works with Cristina Benea. In [1, 2], the authors developed a powerful method, which they called the helicoidal method, that allows to obtain vector-valued inequalities in harmonic analysis. They apply this method to obtain vector-valued inequalities for paraproducts (which can be regarded as bilinear multiplier operators) and the bilinear Hilbert transform. We also refer to [7, 15] where, with different methods, the authors obtain vector-valued inequalities for multilinear multiplier operators. As far as we know, Marcinkiewicz-Zygmund inequalities for multilinear CalderónZygmund operators were not addressed in the weighted case. Also, although it might be possible to derive similar estimates via the helicoidal method, our approach is completely different.

5.1.2. Weighted vector-valued estimates. We state now as a proposition a result that is partially demonstrated in the proof of Theorem 3.1 .

Proposition 5.3. Let $0<p, q_{1}, \ldots, q_{m}<r<2$ or $r=2$ and $0<p, q_{1}, \ldots, q_{m}<\infty$ and, for each $1 \leq i \leq m$, consider $\left\{f_{k_{i}}^{i}\right\}_{k_{i}} \subset L^{q_{i}}\left(\mu_{i}\right)$. Then, there exists a constant $C>0$ such that the following estimates hold.

(i) If $T: L^{q_{1}}\left(\mu_{1}\right) \times \cdots \times L^{q_{m}}\left(\mu_{m}\right) \rightarrow L^{p}(\nu)$ then

$$
\left\|\left(\sum_{k_{1}, \ldots, k_{m}}\left|T\left(f_{k_{1}}^{1}, \ldots, f_{k_{m}}^{m}\right)\right|^{r}\right)^{\frac{1}{r}}\right\|_{L^{p}(\nu)} \leq C\|T\| \prod_{i=1}^{m}\left\|\left(\sum_{k_{i}}\left|f_{k_{i}}^{i}\right|^{r}\right)^{\frac{1}{r}}\right\|_{L^{q_{i}}\left(\mu_{i}\right)} .
$$

(ii) If $T: L^{q_{1}}\left(\mu_{1}\right) \times \cdots \times L^{q_{m}}\left(\mu_{m}\right) \rightarrow L^{p, \infty}(\nu)$ then

$$
\left\|\left(\sum_{k_{1}, \ldots, k_{m}}\left|T\left(f_{k_{1}}^{1}, \ldots, f_{k_{m}}^{m}\right)\right|^{r}\right)^{\frac{1}{r}}\right\|_{L^{p, \infty}(\nu)} \leq C e^{\frac{1}{p}}\|T\|_{\text {weak }} \prod_{i=1}^{m}\left\|\left(\sum_{k_{i}}\left|f_{k_{i}}^{i}\right|^{r}\right)^{\frac{1}{r}}\right\|_{L^{q_{i}\left(\mu_{i}\right)}}
$$

Proof. The inequality (39) was proved in Theorem 3.1 for $1 \leq p, q_{1}, \ldots, q_{m}<r<2$ (see the assertion (a) inside the proof). It is easy to check that, with exactly the same proof, the statement remains valid for $0<p, q_{1}, \ldots, q_{m}<r<2$. The case $r=2$ also follows the same proof, with slight modifications in the involved constants. See the case $r=2<p$ in Lemma 2.4 (see also [18, Thm. 6 (a)]). Then, we only need to prove (ii) and, for this 
purpose, we follow ideas of [18, Thm. $6(\mathrm{~b})]$ where the particular case $r=2$ is addressed. Recall that, for $0<s<p<\infty$,

$$
\|f\|_{L^{p, \infty}(\nu)} \leq \sup _{0<\nu(E)<\infty} \nu(E)^{\frac{1}{p}-\frac{1}{s}}\left(\int_{E}|f|^{s} d \nu\right)^{1 / s} \leq\left(\frac{p}{p-s}\right)^{\frac{1}{s}}\|f\|_{L^{p, \infty}(\nu)} .
$$

Then,

$$
\begin{aligned}
& \left\|\left(\sum_{k_{1}, \ldots, k_{m}}\left|T\left(f_{k_{1}}^{1}, \ldots, f_{k_{m}}^{m}\right)\right|^{r}\right)^{\frac{1}{r}}\right\|_{L^{p, \infty}(\nu)} \leq \\
\leq & \sup _{0<\nu(E)<\infty} \nu(E)^{\frac{1}{p}-\frac{1}{s}}\left(\int_{E}\left(\sum_{k_{1}, \ldots, k_{m}}\left|T\left(f_{k_{1}}^{1}, \ldots, f_{k_{m}}^{m}\right)(\omega)\right|^{r}\right)^{\frac{s}{r}} d \nu(\omega)\right)^{1 / s} \\
= & \sup _{0<\nu(E)<\infty} \nu(E)^{\frac{1}{p}-\frac{1}{s}}\left(\int\left(\sum_{k_{1}, \ldots, k_{m}}\left|\chi_{E}(\omega) T\left(f_{k_{1}}^{1}, \ldots, f_{k_{m}}^{m}\right)(\omega)\right|^{r}\right)^{\frac{s}{r}} d \nu(\omega)\right)^{1 / s} .
\end{aligned}
$$

Now for each measurable set $E$ of positive and finite $\nu$-measure, consider $T_{E}: L^{q_{1}}\left(\mu_{1}\right) \times \cdots \times$ $L^{q_{m}}\left(\mu_{m}\right) \rightarrow L^{s}(\nu)$ defined by

$$
T_{E}\left(f^{1}, \ldots, f^{m}\right)(\omega)=\chi_{E}(\omega) T\left(f^{1}, \ldots, f^{m}\right)(\omega)
$$

(the fact that $T_{E}$ takes values in $L^{s}(\nu)$ follows from (41)). In virtue of the second inequality in (41) we have

$$
\begin{aligned}
\nu(E)^{\frac{1}{p}-\frac{1}{s}}\left\|T_{E}\left(f^{1}, \ldots, f^{m}\right)\right\|_{L^{s}(\nu)} & \leq\left(\frac{p}{p-s}\right)^{\frac{1}{s}}\left\|T\left(f^{1}, \ldots, f^{m}\right)\right\|_{L^{p, \infty}(\nu)} \\
& \leq\left(\frac{p}{p-s}\right)^{\frac{1}{s}}\|T\|_{\text {weak }}\left\|f^{1}\right\|_{L^{q_{1}}\left(\mu_{1}\right)} \cdots\left\|f^{m}\right\|_{L^{q_{m}}\left(\mu_{m}\right)}
\end{aligned}
$$


and, consequently, $\nu(E)^{\frac{1}{p}-\frac{1}{s}}\left\|T_{E}\right\| \leq\left(\frac{p}{p-s}\right)^{\frac{1}{s}}\|T\|_{\text {weak }}$. Going back to (42), we obtain

$$
\begin{aligned}
& \left\|\left(\sum_{k_{1}, \ldots, k_{m}}\left|T\left(f_{1}^{k_{1}}, \ldots, f_{m}^{k_{m}}\right)\right|^{r}\right)^{\frac{1}{r}}\right\|_{L^{p, \infty}(\nu)} \leq \\
\leq & \sup _{0<\nu(E)<\infty} \nu(E)^{\frac{1}{p}-\frac{1}{s}}\left\|\left(\sum_{k_{1}, \ldots, k_{m}}\left|T_{E}\left(f_{k_{1}}^{1}, \ldots, f_{k_{m}}^{m}\right)\right|^{r}\right)^{\frac{1}{r}}\right\|_{s} \\
\leq & \sup _{0<\nu(E)<\infty} \nu(E)^{\frac{1}{p}-\frac{1}{s}} C\left\|T_{E}\right\| \prod_{i=1}^{m}\left\|\left(\sum_{k_{i}}\left|f_{k_{i}}^{i}\right|^{r}\right)^{\frac{1}{r}}\right\|_{L^{q_{i}\left(\mu_{i}\right)}} \quad \text { (by item (i)) } \\
\leq & C\left(\frac{p}{p-s}\right)^{\frac{1}{s}}\|T\|_{\text {weak }} \prod_{i=1}^{m}\left\|\left(\sum_{k_{i}}\left|f_{k_{i}}^{i}\right|^{r}\right)^{\frac{1}{r}}\right\|_{L^{q_{i}\left(\mu_{i}\right)}}
\end{aligned}
$$

and since $0<s<p$ was arbitrary, letting $s \rightarrow 0$ we obtain the desired estimate.

As a consequence of the previous proposition, we obtain the following vector-valued estimates for multilinear Calderón-Zygmund operators which should be compared with those of Theorems 5.1 and 5.2 .

Corollary 5.4. Let $T$ be a multilinear Calderón-Zygmund operator, $1 \leq q_{1}, \ldots, q_{m}<r<2$ (or $r=2$ and $1 \leq q_{1}, \ldots, q_{m}<\infty$ ) and $p>0$ such that $\frac{1}{p}=\frac{1}{q_{1}}+\cdots+\frac{1}{q_{m}}$. Suppose $\vec{w}=\left(w_{1}, \ldots, w_{m}\right)$ satisfies the multilinear $A_{\vec{q}}$ condition and consider $\nu_{\vec{w}}=\prod_{i=1}^{m} w_{i}^{p / q_{i}}$. Then, there exists a constant $C>0$ such that the following estimates hold.

(i) If $q_{i}>1$ for all $1 \leq i \leq m$, then

$$
\left\|\left(\sum_{k_{1}, \ldots, k_{m}}\left|T\left(f_{k_{1}}^{1}, \ldots, f_{k_{m}}^{m}\right)\right|^{r}\right)^{\frac{1}{r}}\right\|_{L^{p}\left(\nu_{\vec{w}}\right)} \leq C\|T\| \prod_{i=1}^{m}\left\|\left(\sum_{k_{i}}\left|f_{k_{i}}^{i}\right|^{r}\right)^{\frac{1}{r}}\right\|_{L^{q_{i}\left(w_{i}\right)}} .
$$

(ii) If at least one $q_{i}=1$, then

$$
\left\|\left(\sum_{k_{1}, \ldots, k_{m}}\left|T\left(f_{k_{1}}^{1}, \ldots, f_{k_{m}}^{m}\right)\right|^{r}\right)^{\frac{1}{r}}\right\|_{L^{p, \infty}\left(\nu_{\vec{w}}\right)} \leq C e^{\frac{1}{p}}\|T\|_{\text {weak }} \prod_{i=1}^{m}\left\|\left(\sum_{k_{i}}\left|f_{k_{i}}^{i}\right|^{r}\right)^{\frac{1}{r}}\right\|_{L^{q_{i}\left(w_{i}\right)}} .
$$

\subsection{Marcinkiewicz-Zygmund inequalities for positive multilinear operators. Fol-} lowing the proof of the Marcinkiewicz-Zygmund inequality for positive linear operators stated in [16, Chapter V.1, Thm. 1.12], we prove Proposition [1.9] which extends this result to the multilinear setting. Recall that a multilinear operator $T: L^{q_{1}}\left(\mu_{1}\right) \times \cdots L^{q_{m}}\left(\mu_{m}\right) \rightarrow L^{p}(\nu)$ is 
positive if $f^{1}, \ldots, f^{m} \geq 0$ implies $T\left(f^{1}, \ldots, f^{m}\right) \geq 0$. It can be seen that if $T$ is positive, then $\left|T\left(f^{1}, \ldots, f^{m}\right)\right| \leq T\left(g^{1}, \ldots, g^{m}\right)$ whenever $\left|f^{1}\right| \leq g^{1}, \ldots,\left|f^{m}\right| \leq g^{m}$.

Proof of Proposition 1.9. It suffices to show that

$$
\left(\sum_{k_{1}, \ldots, k_{m}}\left|T\left(f_{k_{1}}^{1}, \ldots, f_{k_{m}}^{m}\right)(\omega)\right|^{r}\right)^{1 / r} \leq T\left(\left(\sum_{k_{1}=1}^{n_{1}}\left|f_{k_{1}}^{1}\right|^{r}\right)^{1 / r}, \ldots,\left(\sum_{k_{m}=1}^{n_{m}}\left|f_{k_{m}}^{m}\right|^{r}\right)^{1 / r}\right)(\omega)
$$

for any choice of functions $\left\{f_{k_{i}}^{i}\right\}_{k_{i}=1}^{n_{i}} \subset L^{q_{i}}\left(\mu_{i}\right), 1 \leq i \leq m$. The case $r=\infty$ is immediate by the positivity of $T$. Then, we assume $1 \leq r<\infty$ and prove (45) by induction on $m$. The case $m=1$ is proved in [16, Chapter V.1, Thm. 1.12] via a duality argument. Now, let $m \geq 2$ and suppose (45) holds for $m-1$. By duality we know that, given $\mathbf{a}=\left(a_{k_{1} \ldots k_{m}}\right)_{k_{1}, \ldots, k_{m}=1}^{\infty}$,

$$
\|\mathbf{a}\|_{\ell^{r}(\mathbb{N} \times \cdots \times \mathbb{N})}=\sup \left|\sum_{k_{1}, \ldots, k_{m}} a_{k_{1} \ldots k_{m}} b_{k_{1} \ldots k_{m}}\right|
$$

where the supremum is taken over all $\mathbf{b}=\left(b_{k_{1} \ldots k_{m}}\right)_{k_{1}, \ldots, k_{m}}$ such that $\|\mathbf{b}\|_{\ell^{r^{\prime}}(\mathbb{N} \times \cdots \times \mathbb{N})} \leq 1$. Take any $\mathbf{b} \in \ell^{r^{\prime}}(\mathbb{N} \times \cdots \times \mathbb{N})$ and note that

$$
\begin{aligned}
& \left|\sum_{k_{1}, \ldots, k_{m}} T\left(f_{k_{1}}^{1}, \ldots, f_{k_{m}}^{m}\right)(\omega) b_{k_{1} \ldots k_{m}}\right|=\left|\sum_{k_{1}, \ldots, k_{m-1}} T\left(f_{k_{1}}^{1}, \ldots, f_{k_{m}-1}^{m-1}, \sum_{k_{m}} b_{k_{1} \ldots k_{m}} f_{k_{m}}^{m}\right)(\omega)\right| \\
\leq & \sum_{k_{1}, \ldots, k_{m-1}}\left|T\left(f_{k_{1}}^{1}, \ldots, f_{k_{m}-1}^{m-1}, \sum_{k_{m}} b_{k_{1} \ldots k_{m}} f_{k_{m}}^{m}\right)(\omega)\right| \\
\leq & \sum_{k_{1}, \ldots, k_{m-1}} T\left(\left|f_{k_{1}}^{1}\right|, \ldots,\left|f_{k_{m-1}}^{m-1}\right|,\left(\sum_{k_{m}}\left|f_{k_{m}}^{m}\right|^{r}\right)^{1 / r}\right)(\omega)\left(\sum_{k_{m}}\left|b_{k_{1} \ldots k_{m}}\right|^{r^{\prime}}\right)^{1 / r^{\prime}},
\end{aligned}
$$

where the last inequality follows from Hölder's inequality and the positivity of $T$. A repeated application of Hölder's inequality and the induction hypothesis shows that

$$
\begin{aligned}
& \sum_{k_{1}, \ldots, k_{m-1}} T\left(\left|f_{k_{1}}^{1}\right|, \ldots,\left|f_{k_{m-1}}^{m-1}\right|,\left(\sum_{k_{m}}\left|f_{k_{m}}^{m}\right|^{r}\right)^{1 / r}\right)(\omega)\left(\sum_{k_{m}}\left|b_{k_{1} \ldots k_{m}}\right|^{r^{\prime}}\right)^{1 / r^{\prime}} \\
\leq & \left(\sum_{k_{1}, \ldots, k_{m-1}}\left|T\left(\left|f_{k_{1}}^{1}\right|, \ldots,\left|f_{k_{m-1}}^{m-1}\right|,\left(\sum_{k_{m}}\left|f_{k_{m}}^{m}\right|^{r}\right)^{1 / r}\right)(\omega)\right|^{r}\|\mathbf{b}\|_{\ell^{r^{\prime}}(\mathbb{N} \times \cdots \times \mathbb{N})}\right. \\
\leq & T\left(\left(\sum_{k_{1}=1}^{n_{1}}\left|f_{k_{1}}^{1}\right|^{r}\right)^{1 / r}, \ldots,\left(\sum_{k_{m}=1}^{n_{m}}\left|f_{k_{m}}^{m}\right|^{r}\right)^{1 / r}\right)(\omega)\|\mathbf{b}\|_{\ell^{r^{\prime}}(\mathbb{N} \times \cdots \times \mathbb{N})}
\end{aligned}
$$


Hence,

$\left|\sum_{k_{1}, \ldots, k_{m}} T\left(f_{k_{1}}^{1}, \ldots, f_{k_{m}}^{m}\right)(\omega) b_{k_{1} \ldots k_{m}}\right| \leq T\left(\left(\sum_{k_{1}}\left|f_{k_{1}}^{1}\right|^{r}\right)^{1 / r}, \ldots,\left(\sum_{k_{m}}\left|f_{k_{m}}^{m}\right|^{r}\right)^{1 / r}\right)(\omega)\|\mathbf{b}\|_{\ell^{\prime}(\mathbb{N} \times \cdots \times \mathbb{N})}$, and taking supremum over all $\|\mathbf{b}\|_{\ell^{r^{\prime}}(\mathbb{N} \times \cdots \times \mathbb{N})} \leq 1$ we get (45).

As an immediate consequence of Proposition 1.9 and Young's inequality, we have the following vector-valued inequality for the convolution $f * g(x)=\int_{\mathbb{R}} f(x-y) g(y) d y$.

Corollary 5.5. Let $1 \leq q_{1}, q_{2}, p \leq \infty$ satisfying $\frac{1}{q_{1}}+\frac{1}{q_{2}}=\frac{1}{p}+1$ and let $1 \leq r \leq \infty$. Then,

$$
\left\|\left(\sum_{k_{1}, k_{2}}\left|f_{k_{1}}^{1} * f_{k_{2}}^{2}\right|^{r}\right)^{1 / r}\right\|_{L^{p}(\mathbb{R})} \leq\left\|\left(\sum_{k_{1}=1}^{n_{1}}\left|f_{k_{1}}^{1}\right|^{r}\right)^{1 / r}\right\|_{L^{q_{1}(\mathbb{R})}}\left\|\left(\sum_{k_{2}=1}^{n_{2}}\left|f_{k_{2}}^{2}\right|^{r}\right)^{1 / r}\right\|_{L^{q_{2}(\mathbb{R})}}
$$

for any choice of functions $\left\{f_{k_{i}}^{i}\right\}_{k_{i}=1}^{n_{i}} \subset L^{q_{i}}(\mathbb{R}), 1 \leq i \leq 2$.

The inequality (8) should be compared with [13, Thm. 6.2], where it is proved that, given a positive $m$-linear operator $T: X_{1} \times \cdots \times X_{m} \rightarrow Y$ between Banach lattices and $1 \leq r_{1}, \ldots, r_{m}, r \leq \infty$ such that $\frac{1}{r}=\frac{1}{r_{1}}+\cdots+\frac{1}{r_{m}}$, we have

$$
\left\|\left(\sum_{k}\left|T\left(x_{k}^{1}, \ldots, x_{k}^{m}\right)\right|^{r}\right)^{1 / r}\right\|_{Y} \leq\|T\| \prod_{i=1}^{m}\left\|\left(\sum_{k=1}^{n}\left|x_{k}^{i}\right|^{r_{i}}\right)^{1 / r_{i}}\right\|_{X_{i}}
$$

for any choice of sequences $\left\{x_{k}^{i}\right\}_{k=1}^{n} \subset X_{i}, 1 \leq i \leq m$. As a consequence, if $1 \leq q_{1}, q_{2}, p \leq \infty$ satisfy $\frac{1}{q_{1}}+\frac{1}{q_{2}}=\frac{1}{p}+1$ and $1 \leq r_{1}, r_{2}, r \leq \infty$ are such that $\frac{1}{r}=\frac{1}{r_{1}}+\frac{1}{r_{2}}$, then

$$
\left\|\left(\sum_{k=1}^{n}\left|f_{k}^{1} * f_{k}^{2}\right|^{r}\right)^{1 / r}\right\|_{L^{p}(\mathbb{R})} \leq\left\|\left(\sum_{k=1}^{n}\left|f_{k}^{1}\right|^{r_{1}}\right)^{1 / r_{1}}\right\|_{L^{q_{1}(\mathbb{R})}}\left\|\left(\sum_{k=1}^{n}\left|f_{k}^{2}\right|^{r_{2}}\right)^{1 / r_{2}}\right\|_{L^{q_{2}(\mathbb{R})}}
$$

for any choice of functions $\left\{f_{k}^{i}\right\}_{k=1}^{n} \subset L^{q_{i}}(\mathbb{R}), 1 \leq i \leq 2$.

Acknowledgements. The authors wish to thank C. Muscalu for his valuable comments regarding this work.

\section{REFERENCES}

[1] Benea C. and Muscalu C.. Multiple vector valued inequalities via the helicoidal method. Anal. PDE, 9: 1931-1988, 2016.

[2] Benea C. and Muscalu C.. Quasi-Banach valued inequalities via the helicoidal method. Preprint, arXiv: 1609.01090.

[3] Bergh J. and Löfström J.. Interpolation Spaces. An Introduction. Grund. Math. Wiss. 223, SpringerVerlag, Berlin, 1976. 
[4] Boas H.. Majorant Series. J. Korean Math. Soc., 37(2): 321-337, 2000.

[5] Bombal F., Pérez-García D. and Villanueva I.. Multilinear extensions of Grothendieck's theorem. Quart. J. Math., 55(4): 441-450, 2004.

[6] Bohnenblust H. F. and Hille E.. On the absolute convergence of Dirichlet series. Ann. of Math., 32(3): 600-622, 1931.

[7] Culiuc A., Di Plinio F. and Ou Y.. Domination of multilinear singular integrals by positive sparse forms. Preprint, arXiv: 1603.05317.

[8] Cruz-Uribe D., Martell J.M. and Pérez C.. Extrapolation from $A_{\infty}$ weights and applications. J. Funct. Anal., 213(2): 412-439, 2004.

[9] Curbera G., García-Cuerva J., Martell J.M., Pérez C.. Extrapolation with weights, rearrangementinvariant function spaces, modular inequalities and applications to singular integrals. Adv. Math., 203: 256-318, 2006.

[10] Davie A.M.. Quotient algebras of uniform algebras. J. London Math. Soc., 7(2): 31-40, 1973.

[11] Defant A. and Floret K.. Tensor Norms and Operator Ideals. North-Holland Math. Stud. 176, North Holland, 1993.

[12] Defant A. and Junge M.. Best constants and asymptotics of Marcinkiewicz-Zygmund inequalities. Studia Math., 125(3): 271-287, 1997.

[13] Defant A. and Mastyło M.. Interpolation of Fremlin tensor products and Schur factorization of matrices. J. Funct. Anal., 262: 3981-3999, 2012.

[14] Defant A. and Sevilla-Peris P.. A new multilinear insight on Littlewood's 4/3-inequality. J. Funct. Anal., 256(5): 1642-1664, 2009.

[15] Di Plinio F. and Ou Y.. Banach-valued multilinear singular integrals. Preprint, arXiv: 1506.05827. To appear in Indiana Univ. Math. J..

[16] García-Cuerva J. and Rubio de Francia J.L.. Weighted norm inequalities and related topics. NorthHolland Math. Stud. 116, North-Holland, 1985.

[17] Gasch J. and Maligranda L.. On vector-valued inequalities of Marcinkiewicz-Zygmund, Herz and Krivine type. Math. Nachr., 167: 95-129, 1994.

[18] Grafakos L. and Martell J.M.. Extrapolation of weighted norm inequalities for multivariable operators and applications. J. Geom. Anal., 14(1): 19-46, 2004.

[19] Grafakos L. and Torres R.H.. Multilinear Calderón-Zygmund theory. Adv. Math., 165(1): 124-164, 2002.

[20] Grafakos L. and Torres R.H.. Maximal operator and weighted norm inequalities for multilinear singular integrals. Indiana Univ. Math. J., 51(5): 1261-1276, 2002.

[21] Herz C.. Theory of $p$-spaces with an application to convolution operators. Trans. Amer. Math. Soc., 154: 69-82, 1971.

[22] Kaijser S.. Some results in the metric theory of tensor products. Studia Math. 63(2): 157-170, 1978.

[23] Lerner A., Ombrosi S., Pérez C., Torres R. and Trujillo-González R.. New maximal functions and multiple weights for the multilinear Calderón-Zygmund theory. Adv. Math., 220(4): 1222-1264, 2009.

[24] Marcinkiewicz J. and Zygmund A.. Quelques inégalités pour les opérations linéaires. Fund. Math., 32: 113-121, 1939.

[25] Pérez C. and Torres R.H.. Sharp maximal function estimates for multilinear singular integrals. Contemp. Math., 320: 323-333, 2003.

[26] Pietsch A.. Operator Ideals. North-Holland, Amsterdam, 1980.

[27] Quefflec H.. H. Bohr's vision of ordinary Dirichlet series; old and new results. J. Anal., 3: 43-60, 1995.

Departamento de Matemática - Pab i, Facultad de Cs. Exactas y Naturales, Universidad de Buenos Aires, (1428) Buenos Aires, Argentina and IMAS-CONiCET

E-mail address: dcarando@dm.uba.ar 
Instituto Balseiro, Universidad Nacional de Cuyo - C.N.E.A. and Departamento de Matemática, Centro Regional Universitario Bariloche, Universidad Nacional del Comahue, (8400) San Carlos de Bariloche, Argentina

E-mail address: martin.mazzitelli@crub.uncoma.edu.ar

Departamento de Matemática, Universidad Nacional del Sur, (8000) Bahía Blanca, ArGENTINA AND INMABB-CONICET

E-mail address: sombrosi@uns.edu.ar 